This is an electronic reprint of the original article. This reprint may differ from the original in pagination and typographic detail.

Author(s): Heikkinen, Toni; Koskela, Pekka; Tuominen, Heli

Title: $\quad$ Approximation and Quasicontinuity of Besov and Triebel-Lizorkin Functions

Year: $\quad 2017$

Version:

Please cite the original version:

Heikkinen, T., Koskela, P., \& Tuominen, H. (2017). Approximation and Quasicontinuity of Besov and Triebel-Lizorkin Functions. Transactions of the American Mathematical Society, 369(5), 3547-3573.

https://doi.org/10.1090/tran/6886

All material supplied via JYX is protected by copyright and other intellectual property rights, and duplication or sale of all or part of any of the repository collections is not permitted, except that material may be duplicated by you for your research use or educational purposes in electronic or print form. You must obtain permission for any other use. Electronic or print copies may not be offered, whether for sale or otherwise to anyone who is not an authorised user. 


\title{
APPROXIMATION AND QUASICONTINUITY OF BESOV AND TRIEBEL-LIZORKIN FUNCTIONS
}

\author{
TONI HEIKKINEN, PEKKA KOSKELA AND HELI TUOMINEN
}

\begin{abstract}
We show that, for $0<s<1,0<p, q<\infty$, Hajłasz-Besov and Hajłasz-Triebel-Lizorkin functions can be approximated in the norm by discrete median convolutions. This allows us to show that, for these functions, the limit of medians,

$$
\lim _{r \rightarrow 0} m_{u}^{\gamma}(B(x, r))=u^{*}(x),
$$

exists quasieverywhere and defines a quasicontinuous representative of $u$. The above limit exists quasieverywhere also for Hajłasz functions $u \in M^{s, p}, 0<s \leq 1$, $0<p<\infty$, but approximation of $u$ in $M^{s, p}$ by discrete (median) convolutions is not in general possible.
\end{abstract}

\section{INTRODUCTION}

Smooth functions are dense in the classical Sobolev space $W^{1, p}\left(\mathbb{R}^{n}\right)$, as can be seen by convolution approximation. This fact implies that each Sobolev function has a quasicontinuous representative and can be defined everywhere, except for a set of $p$-capacity zero, in terms of limits of integral averages. Indeed, by the Lebesgue differentiation theorem, almost every point $x \in \mathbb{R}^{n}$ is a Lebesgue point,

$$
\lim _{r \rightarrow 0} \frac{1}{|B(x, r)|} \int_{B(x, r)}|u(y)-u(x)| d y=0,
$$

which implies that

$$
\lim _{r \rightarrow 0} \frac{1}{|B(x, r)|} \int_{B(x, r)} u(y) d y=u(x)
$$

for such $x$ when $u$ is locally integrable. For $u \in W^{1, p}\left(\mathbb{R}^{n}\right)$, the set of exceptional points where (1.1) fails, is smaller, of $p$-capacity zero. The central tools to prove this fact are density of smooth functions and capacitary weak-type estimates for the Hardy-Littlewood maximal function, see for example [11], [47]. Part of this argument is almost axiomatic and generalizes to other function spaces with suitable modifications.

In this paper, we study approximation of Besov and Triebel-Lizorkin functions and the consequent existence of Lebesgue points in a metric space $X$ equipped with a doubling measure $\mu$. Because of lack of a linear structure, one cannot use the

2010 Mathematics Subject Classification. 46E35, $43 \mathrm{~A} 85$.

Key words and phrases. Besov space, Triebel-Lizorkin space, fractional Sobolev space, metric measure space, median, quasicontinuity. 
usual convolution approach. Hence we employ a discrete convolution, which for a locally integrable function $u$ at the scale $r$ is defined as

$$
u_{r}=\sum_{i} \frac{1}{\left|B_{i}\right|} \int_{B_{i}} u d \mu \varphi_{i}
$$

where $\left\{B_{i}\right\}_{i}$ is a covering of $X$ by balls of radius $r$ and $\left\{\varphi_{i}\right\}_{i}$ is a partition of unity associated to the covering $\left\{B_{i}\right\}_{i}$, see Section 2 for details. Discrete convolutions have been standard tools in analysis in doubling metric spaces starting from the works [8] and [33].

If our function $u$ fails to be locally integrable, as can happen with Besov and Triebel-Lizorkin spaces for small indices $p$ and $q$, we need to modify the definition of our discrete convolution and use medians instead of integral averages. For $0<$ $\gamma \leq 1 / 2$, the $\gamma$-median of a measurable function $u: X \rightarrow \overline{\mathbb{R}}$ over a set $A$ of finite measure is

$$
m_{u}^{\gamma}(A)=\inf \{a \in \mathbb{R}: \mu(\{x \in A: u(x)>a\})<\gamma \mu(A)\} .
$$

Median values and median maximal functions have already been studied and used in different problems of analysis for quite some time, see [12], [14], [15], [20], [23], [24], [25], [31], 32], 338], [43] and [46].

We consider the Hajłasz-Besov spaces $N_{p, q}^{s}(X)$ and Hajłasz-Triebel-Lizorkin spaces $M_{p, q}^{s}(X)$ which were recently introduced in [30] and studied for example in [15], [21], [22] and [20]. These spaces consist of those $L^{p}$-functions $u$ that have a fractional $s$-gradient with finite mixed $l^{q}\left(L^{p}(X)\right)$ - or $L^{p}\left(X, l^{q}\right)$-norm. A sequence $\left(g_{k}\right)_{k \in \mathbb{Z}}$ of measurable functions $g_{k}: X \rightarrow[0, \infty]$ is a fractional $s$-gradient of $u$ if it satisfies the Hajłasz-type pointwise inequality

$$
|u(x)-u(y)| \leq d(x, y)^{s}\left(g_{k}(x)+g_{k}(y)\right)
$$

for all $k \in \mathbb{Z}$ and almost all $x, y \in X$ satisfying $2^{-k-1} \leq d(x, y)<2^{-k}$, see Section 2 for details. As usual, the homogeneous versions $\dot{\dot{N}_{p, q}^{s}}(X)$ and $\dot{M}_{p, q}^{s}(X)$ of our Hajłasz-Besov and Hajłasz-Triebel-Lizorkin spaces are defined by relaxing the $L^{p}$ integrability assumption to mere measurability and finiteness almost everywhere.

In the Euclidean case, $N_{p, q}^{s}\left(\mathbb{R}^{n}\right)=B_{p, q}^{s}\left(\mathbb{R}^{n}\right)$ and $M_{p, q}^{s}\left(\mathbb{R}^{n}\right)=F_{p, q}^{s}\left(\mathbb{R}^{n}\right)$ for all $0<p<\infty, 0<q \leq \infty, 0<s<1$, where $B_{p, q}^{s}\left(\mathbb{R}^{n}\right)$ and $F_{p, q}^{s}\left(\mathbb{R}^{n}\right)$ are the Besov and Triebel-Lizorkin spaces defined via an $L^{p}$-modulus of smoothness, see [15]. Recall also that the Fourier analytic approach gives the same spaces when $p>n /(n+s)$ in the Besov case and when $p, q>n /(n+s)$ in the Triebel-Lizorkin case. Hence, for such indices, our results cover also the case of classical Besov and Triebel-Lizorkin spaces.

Our first main result shows that locally Lipschitz functions are dense in $N_{p, q}^{s}(X)$ and in $M_{p, q}^{s}(X)$.

Theorem 1.1. Let $0<\gamma \leq 1 / 2,0<s<1,0<p, q<\infty$ and $u \in \dot{N}_{p, q}^{s}(X)$. Then the discrete $\gamma$-median convolution approximations $u_{i}=u_{2^{-i}}^{\gamma}$ converge to $u$ in $N_{p, q}^{s}(X)$ as $i \rightarrow \infty$. The same result holds with $N_{p, q}^{s}$ replaced by $M_{p, q}^{s}$. 
Concerning earlier approximation results for Besov and Triebel-Lizorkin functions, recall first that smooth functions are dense in the classical Besov and TriebelLizorkin spaces for finite $p, q$ but not necessarily otherwise, see 44 for precise statements. For metric spaces equipped with doubling measure, the density of Lipschitz functions in the homogenous spaces $\dot{M}_{p, q}^{s}(X)$ for $Q /(Q+s)<p, q<\infty$ has been established in [6]. For the so-called RD-spaces, the density follows already from [18, Prop 5.21] for $\dot{N}_{p, q}^{s}(X)$ with $Q /(Q+s)<p<\infty, 0<q<\infty$, and for $\dot{M}_{p, q}^{s}(X)$ with $Q /(Q+s)<p, q<\infty$. Moreover, the density in $N_{p, p}^{Q / p}(X), p>Q$, for Ahlfors $Q$-regular metric spaces $X$ has been proved in [7], [9].

Our second main results says that the limit of medians exists outside a set of capacity zero. We say that such points are generalized Lebesgue points of $u$.

Theorem 1.2. Let $0<s<1,0<p, q<\infty$ and $\mathcal{F} \in\left\{N_{p, q}^{s}, M_{p, q}^{s}\right\}$ or $0<s \leq 1$, $0<p<\infty$ and $\mathcal{F}=M_{p, \infty}^{s}=M^{s, p}$. Let $u \in \dot{\mathcal{F}}(X)$. Then there exists a set $E \subset X$ with $C_{\mathcal{F}}(E)=0$ such that the limit

$$
\lim _{r \rightarrow 0} m_{u}^{\gamma}(B(x, r))=u^{*}(x)
$$

exists for every $x \in X \backslash E$ and $0<\gamma \leq 1 / 2$. Moreover, $u^{*}$ is an $\mathcal{F}$-quasicontinuous representative of $u$.

The existence of Lebesgue points for Besov and Triebel-Lizorkin functions in the Euclidean setting has been studied in [3], [10], [19], [34], [37], but we are not aware of earlier results in the metric setting.

The paper is organized as follows. We present the notation and definitions used in the paper in Section 2. We discuss $\gamma$-medians and their basic properties and define two maximal functions related to $\gamma$-medians. The discrete version is a modification of the discrete maximal function used for locally integrable functions and comparable with the maximal function defined via medians over balls.

We prove Theorem 1.1, convergence of discrete median convolutions in the Hajłasz-Besov and Hajłasz-Triebel-Lizorkin norms, in Sections 3 and 4. In the former section, the proof is given in the setting of a doubling metric space. In the latter section, we give a a substantially simpler proof under the additional assumption that spheres are nonempty. We also give a simple example which shows that discrete (median) convolution approximations of a Hajłasz-Sobolev function $u$ do not necessarily converge to $u$ in the norm.

In Section 5, we show that also the discrete convolution approximations converge in the Hajłasz-Besov and Hajłasz-Triebel-Lizorkin norms if $Q /(Q+s)<p<\infty$ (where $Q$ is the doubling dimension) in the Besov case and $Q /(Q+s)<q<\infty$ in the Triebel-Lizorkin case.

In Section 6, we discuss capacities connected to the Hajłasz-Besov and HajłaszTriebel-Lizorkin spaces. These capacities are not necessarily subadditive, but they satisfy the inequality $\operatorname{Cap}\left(\cup_{i} E_{i}\right)^{r} \leq c \sum_{i} \operatorname{Cap}\left(E_{i}\right)^{r}$ for all sets $E_{i} \subset X, i \in \mathbb{N}$ for $r=\min \{1, q / p\}$ (compare this with the Aoki-Rolewicz Theorem for triangle inequality for quasi norms). 
Our second main result, Theorem 1.2 is proved in Section 7 . The central tools used in the proof are Theorems [7.6 and 7.7] which show that the discrete median maximal operator is bounded on Hajłasz-Besov and Hajłasz-Triebel-Lizorkin spaces and that a capacitary weak-type estimate holds for the median maximal function.

The final section deals with the existence of the classical Lebesgue points defined as limits of integral averages over balls. We show that counterparts of results of Section 7 hold for locally integrable functions for suitable parameters of the space. In particular, Corollary 8.5 gives new results for the existence Lebesgue points of functions in fractional Hajłasz-Sobolev spaces $M^{s, p}(X)$ for parameters $0<s \leq 1$ and $Q /(Q+s)<p<\infty$.

\section{Preliminaries}

2.1. Basic assumptions. In this paper, $X=(X, d, \mu)$ is a metric measure space equipped with a metric $d$ and a Borel regular, doubling outer measure $\mu$, for which the measure of every ball is positive and finite. The doubling property means that there exists a constant $c_{d}>0$, called the doubling constant, such that

$$
\mu(B(x, 2 r)) \leq c_{d} \mu(B(x, r))
$$

for every ball $B(x, r)=\{y \in X: d(y, x)<r\}$, where $x \in X$ and $r>0$.

The doubling condition gives an upper bound for the dimension of $X$ because there is a constant $c=c\left(c_{d}\right)$ such that

$$
\frac{\mu(B(y, r))}{\mu(B(x, R))} \geq c\left(\frac{r}{R}\right)^{Q}
$$

for every $0<r \leq R$ and $y \in B(x, R)$ with $Q=\log _{2} c_{d}$. Below $Q$ refers to this dimension.

The integral average of a locally integrable function $u$ over a set $A$ of positive and finite measure is

$$
u_{A}=f_{A} u d \mu=\frac{1}{\mu(A)} \int_{A} u d \mu .
$$

By $\chi_{E}$, we denote the characteristic function of a set $E \subset X$ and by $\overline{\mathbb{R}}$, the extended real numbers $[-\infty, \infty] . L^{0}(X)$ is the set of all measurable, almost everywhere finite functions $u: X \rightarrow \overline{\mathbb{R}}$. In general, $C$ and $c$ are positive constants whose values are not necessarily same at each occurrence. When we want to stress that the constant depends on other constants or parameters $a, b, \ldots$, we write $C=C(a, b, \ldots)$.

2.2. Hajłasz spaces, Hajłasz-Besov and Hajłasz-Triebel-Lizorkin spaces. Among several definitions for Besov and Triebel-Lizorkin spaces in metric measure spaces, we use the definitions given by pointwise inequalities in [30]. This definition is motivated by the Hajłasz-Sobolev spaces $M^{s, p}(X)$, defined for $s=1, p \geq 1$ in [17] and for fractional scales in [45].

Definition 2.1. Let $0<s<\infty$. A measurable function $g \geq 0$ is an s-gradient of a function $u \in L^{0}(X)$ if there exists a set $E \subset X$ with $\mu(E)=0$ such that for all $x, y \in X \backslash E$,

$$
|u(x)-u(y)| \leq d(x, y)^{s}(g(x)+g(y))
$$


The collection of all $s$-gradients of $u$ is denoted by $\mathcal{D}^{s}(u)$.

Let $0<p \leq \infty$. The homogeneous Hajtasz space $\dot{M}^{s, p}(X)$ consists of those measurable functions $u$ for which

$$
\|u\|_{\dot{M}^{s, p}(X)}=\inf _{g \in \mathcal{D}^{s}(u)}\|g\|_{L^{p}(X)}
$$

is finite. The Hajtasz space $M^{s, p}(X)$ is $\dot{M}^{s, p}(X) \cap L^{p}(X)$ equipped with the norm

$$
\|u\|_{M^{s, p}(X)}=\|u\|_{L^{p}(X)}+\|u\|_{\dot{M}^{s, p}(X)} .
$$

Recall that for $p>1, M^{1, p}\left(\mathbb{R}^{n}\right)=W^{1, p}\left(\mathbb{R}^{n}\right)$, see [17], whereas for $n /(n+1)<$ $p \leq 1, M^{1, p}\left(\mathbb{R}^{n}\right)$ coincides with the Hardy-Sobolev space $H^{1, p}\left(\mathbb{R}^{n}\right)$ by [29, Thm 1].

Definition 2.2. Let $0<s<\infty$. A sequence of nonnegative measurable functions $\left(g_{k}\right)_{k \in \mathbb{Z}}$ is a fractional s-gradient of a function $u \in L^{0}(X)$, if there exists a set $E \subset X$ with $\mu(E)=0$ such that

$$
|u(x)-u(y)| \leq d(x, y)^{s}\left(g_{k}(x)+g_{k}(y)\right)
$$

for all $k \in \mathbb{Z}$ and all $x, y \in X \backslash E$ satisfying $2^{-k-1} \leq d(x, y)<2^{-k}$. The collection of all fractional $s$-gradients of $u$ is denoted by $\mathbb{D}^{s}(u)$.

The next two lemmas for fractional $s$-gradients follow easily from the definition. The corresponding results for 1-gradients have been proved in [27, Lemmas 2.4] and [26, Lemma 2.6]. We leave the proofs for the reader.

Lemma 2.3. Let $u, v \in L^{0}(X),\left(g_{k}\right)_{k \in Z} \in \mathbb{D}^{s}(u)$ and $\left(h_{k}\right)_{k \in Z} \in \mathbb{D}^{s}(v)$. Then the sequence $\left(\max \left\{g_{k}, h_{k}\right\}\right)_{k \in \mathbb{Z}}$ is a fractional s-gradient of the functions $\max \{u, v\}$ and $\min \{u, v\}$.

Lemma 2.4. Let $u_{i} \in L^{0}(X)$ and $\left(g_{i, k}\right)_{k \in \mathbb{Z}} \in \mathbb{D}^{s}\left(u_{i}\right), i \in \mathbb{N}$. Let $u=\sup _{i \in \mathbb{N}} u_{i}$ and $\left(g_{k}\right)_{k \in \mathbb{Z}}=\left(\sup _{i \in \mathbb{N}} g_{i, k}\right)_{k \in \mathbb{Z}}$. If $u \in L^{0}(X)$, then $\left(g_{k}\right)_{k \in \mathbb{Z}} \in \mathbb{D}^{s}(u)$.

For $0<p, q \leq \infty$ and a sequence $\left(f_{k}\right)_{k \in \mathbb{Z}}$ of measurable functions, we define

$$
\left\|\left(f_{k}\right)_{k \in \mathbb{Z}}\right\|_{L^{p}\left(X, l^{q}\right)}=\|\|\left(f_{k}\right)_{k \in \mathbb{Z}}\left\|_{l^{q}}\right\|_{L^{p}(X)}
$$

and

where

$$
\left\|\left(f_{k}\right)_{k \in \mathbb{Z}}\right\|_{l^{q}\left(L^{p}(X)\right)}=\left\|\left(\left\|f_{k}\right\|_{L^{p}(X)}\right)_{k \in \mathbb{Z}}\right\|_{l^{q}},
$$

$$
\left\|\left(f_{k}\right)_{k \in \mathbb{Z}}\right\|_{l^{q}}= \begin{cases}\left(\sum_{k \in \mathbb{Z}}\left|f_{k}\right|^{q}\right)^{1 / q}, & \text { if } 0<q<\infty, \\ \sup _{k \in \mathbb{Z}}\left|f_{k}\right|, & \text { if } q=\infty .\end{cases}
$$

Definition 2.5. Let $0<s<\infty$ and $0<p, q \leq \infty$. The homogeneous HajtaszTriebel-Lizorkin space $\dot{M}_{p, q}^{s}(X)$ consists of those functions $u \in L^{0}(X)$, for which the (semi)norm

$$
\|u\|_{\dot{M}_{p, q}^{s}(X)}=\inf _{\left(g_{k}\right) \in \mathbb{D}^{s}(u)}\left\|\left(g_{k}\right)\right\|_{L^{p}\left(X, l^{q}\right)}
$$

is finite. The Hajtasz-Triebel-Lizorkin space $M_{p, q}^{s}(X)$ is $\dot{M}_{p, q}^{s}(X) \cap L^{p}(X)$ equipped with the norm

$$
\|u\|_{M_{p, q}^{s}(X)}=\|u\|_{L^{p}(X)}+\|u\|_{\dot{M}_{p, q}^{s}(X)} .
$$


Notice that $M_{p, \infty}^{s}(X)=M^{s, p}(X)$, see [30, Prop. 2.1] for a simple proof of this fact.

Similarly, the homogeneous Hajtasz-Besov space $\dot{N}_{p, q}^{s}(X)$ consists of those functions $u \in L^{0}(X)$, for which

$$
\|u\|_{\dot{N}_{p, q}^{s}(X)}=\inf _{\left(g_{k}\right) \in \mathbb{D}^{s}(u)}\left\|\left(g_{k}\right)\right\|_{l^{q}\left(L^{p}(X)\right)}
$$

is finite, and the Hajtasz-Besov space $N_{p, q}^{s}(X)$ is $\dot{N}_{p, q}^{s}(X) \cap L^{p}(X)$ equipped with the norm

$$
\|u\|_{N_{p, q}^{s}(X)}=\|u\|_{L^{p}(X)}+\|u\|_{\dot{N}_{p, q}^{s}(X)} .
$$

For $0<s<1,0<p, q \leq \infty$, the spaces $N_{p, q}^{s}\left(\mathbb{R}^{n}\right)$ and $M_{p, q}^{s}\left(\mathbb{R}^{n}\right)$ coincide with the classical Besov and Triebel-Lizorkin spaces defined via differences ( $L^{p}$-modulus of smoothness), see [15].

When $0<p<1$ or $0<q<1$, the (semi)norms defined above are actually quasi(semi)norms, but for simplicity we call them, as well as other quasi-seminorms in this paper, just norms.

2.3. Inequalities. We will frequently use the elementary inequality

$$
\sum_{i \in \mathbb{Z}} a_{i} \leq\left(\sum_{i \in \mathbb{Z}} a_{i}^{\beta}\right)^{1 / \beta},
$$

which holds whenever $a_{i} \geq 0$ for all $i$ and $0<\beta \leq 1$.

By (2.3), if $0<p<1$ and by the Minkowski inequality, if $p \geq 1$, we have

$$
\left\|\sum_{i \in \mathbb{Z}} f_{i}\right\|_{L^{p}(X)}^{\tilde{p}} \leq \sum_{i \in \mathbb{Z}}\left\|f_{i}\right\|_{L^{p}(X)}^{\tilde{p}}
$$

where $\tilde{p}=\min \{1, p\}$.

The Hölder inequality and (2.3) easily imply the following lemma, which is used to estimate the norms of fractional gradients.

Lemma 2.6 ([20], Lemma 3.1). Let $1<a<\infty, 0<b<\infty$ and $c_{k} \geq 0, k \in \mathbb{Z}$. There exists a constant $C=C(a, b)$ such that

$$
\sum_{k \in \mathbb{Z}}\left(\sum_{j \in \mathbb{Z}} a^{-|j-k|} c_{j}\right)^{b} \leq C \sum_{j \in \mathbb{Z}} c_{j}^{b} .
$$

The Fefferman-Stein vector-valued maximal theorem (see [13], [16], [40]) states that, for $1<p, q \leq \infty$, there exists a constant $C=C\left(c_{d}, p, q\right)$ such that

$$
\left\|\left(\mathcal{M} u_{k}\right)\right\|_{L^{p}\left(X ; l^{q}\right)} \leq C\left\|\left(u_{k}\right)\right\|_{L^{p}\left(X ; l^{q}\right)}
$$

for every $\left(u_{k}\right)_{k \in \mathbb{Z}} \in L^{p}\left(X ; l^{q}\right)$. Here $\mathcal{M}$ is the usual Hardy-Littlewood maximal operator. 
2.4. $\gamma$-median. Recall from the introduction that for $0<\gamma \leq 1 / 2$, the $\gamma$-median of a measurable function $u: X \rightarrow \overline{\mathbb{R}}$ over a set $A$ of finite measure is

$$
m_{u}^{\gamma}(A)=\inf \{a \in \mathbb{R}: \mu(\{x \in A: u(x)>a\})<\gamma \mu(A)\},
$$

and note that if $u \in L^{0}(A)$ and $0<\mu(A)<\infty$, then $m_{u}^{\gamma}(A)$ is finite.

In the following lemma, we list some basic properties of the $\gamma$-median. We leave the quite straightforward proof for the reader, who can also look at [38] where most of the properties are proved in the Euclidean setting. Properties (a), (b), (d), (f), (g) and (h) follow from [38, Propositions 1.1 and 1.2] and (i) and (j) from [38, Theorem 2.1]. The remaining properties (c) and (e) follow immediately from the definition.

Lemma 2.7. The $\gamma$-median has the following properties:

(a) If $\gamma \leq \gamma^{\prime}$, then $m_{u}^{\gamma}(A) \geq m_{u}^{\gamma^{\prime}}(A)$.

(b) If $u \leq v$ almost everywhere, then $m_{u}^{\gamma}(A) \leq m_{v}^{\gamma}(A)$.

(c) If $A \subset B$ and $\mu(B) \leq C \mu(A)$, then $m_{u}^{\gamma}(A) \leq m_{u}^{\gamma / C}(B)$.

(d) If $c \in \mathbb{R}$, then $m_{u}^{\gamma}(A)+c=m_{u+c}^{\gamma}(A)$.

(e) If $c>0$, then $m_{c u}^{\gamma}(A)=c m_{u}^{\gamma}(A)$.

(f) $\left|m_{u}^{\gamma}(A)\right| \leq m_{|u|}^{\gamma}(A)$.

(g) $m_{u+v}^{\gamma}(A) \leq m_{u}^{\gamma / 2}(A)+m_{v}^{\gamma / 2}(A)$.

(h) For every $p>0$,

$$
m_{|u|}^{\gamma}(A) \leq\left(\gamma^{-1} f_{A}|u|^{p} d \mu\right)^{1 / p} .
$$

(i) If $u$ is continuous, then for every $x \in X$,

$$
\lim _{r \rightarrow 0} m_{u}^{\gamma}(B(x, r))=u(x) .
$$

(j) If $u \in L^{0}(X)$, then there exists a set $E$ with $\mu(E)=0$ such that

$$
\begin{aligned}
& \qquad \lim _{r \rightarrow 0} m_{u}^{\gamma}(B(x, r))=u(x) \\
& \text { for every } 0<\gamma \leq 1 / 2 \text { and } x \in X \backslash E .
\end{aligned}
$$

Property (j) above says that medians over small balls behave like integral averages of locally integrable functions at Lebesgue points.

Definition 2.8. Let $u \in L^{0}(A)$. A point $x$ is a generalized Lebesgue point of $u$, if

$$
\lim _{r \rightarrow 0} m_{u}^{\gamma}(B(x, r))=u(x)
$$

for all $0<\gamma \leq 1 / 2$.

2.5. Maximal functions. We use two maximal operators defined using medians. The first one is defined in the usual way by taking a supremum of medians over balls and the second one is a discrete version defined using discrete convolutions.

Definition 2.9. Let $0<\gamma \leq 1 / 2$. The $\gamma$-median maximal function of a function $u \in L^{0}(X)$ is $\mathcal{M}^{\gamma} u: X \rightarrow \overline{\mathbb{R}}$

$$
\mathcal{M}^{\gamma} u(x)=\sup _{r>0} m_{|u|}^{\gamma}(B(x, r)) .
$$


Note that by Lemma $2.7(\mathrm{~h})$ and $(\mathrm{j})$, for any $p>0$,

$$
u(x) \leq \mathcal{M}^{\gamma} u(x) \leq\left(\gamma^{-1} \mathcal{M} u^{p}(x)\right)^{1 / p}
$$

for almost all $x \in X$. It follows from the Hardy-Littlewood maximal theorem that, for any $p>0$, there exists a constant $C=C\left(\gamma, p, c_{d}\right)$ such that

$$
\left\|\mathcal{M}^{\gamma} u\right\|_{L^{p}(X)} \leq C\|u\|_{L^{p}(X)},
$$

for every $u \in L^{p}(X)$. More generally, (2.6) and the Fefferman-Stein maximal theorem, (2.5), imply that, for all $0<p, q \leq \infty$, there exists a constant $C=C\left(\gamma, p, q, c_{d}\right)$ such that

$$
\left\|\left(\mathcal{M}^{\gamma} u_{k}\right)\right\|_{L^{p}\left(X ; l^{q}\right)} \leq C\left\|\left(u_{k}\right)\right\|_{L^{p}\left(X ; l^{q}\right)}
$$

for every $\left(u_{k}\right)_{k \in \mathbb{Z}} \in L^{p}\left(X ; l^{q}\right)$.

For the discrete maximal function, we first recall a definition of a discrete convolution. Discrete convolutions are basic tools in harmonic analysis in homogeneous spaces, see for example [8] and [33. The discrete maximal function, which can be seen as a smooth version of the Hardy-Littlewood maximal function, was introduced in [26].

Let $r>0$ and let $\left\{B_{i}\right\}_{i \in I}$, where $I \subset \mathbb{N}$, be a covering of $X$ by balls of radius $r$ such that

$$
\sum_{i \in I} \chi_{2 B_{i}} \leq C\left(c_{d}\right)
$$

For such a covering, there exist $C / r$-Lipschitz functions $\varphi_{i}, i \in I$, and a constant $C=C\left(c_{d}\right)$ such that $0 \leq \varphi_{i} \leq 1, \varphi_{i}=0$ outside $2 B_{i}$ and $\varphi_{i} \geq C^{-1}$ on $B_{i}$ for all $i$ and $\sum_{i \in I} \varphi_{i}=1$. Such a collection of functions is called a partition of unity subordinate to the covering $\left\{B_{i}\right\}_{i \in I}$. A discrete convolution of a locally integrable function $u$ at the scale $r$ is

$$
u_{r}=\sum_{i \in I} u_{B_{i}} \varphi_{i}
$$

and the discrete maximal function of $u$ is $\mathcal{M}^{*} u: X \rightarrow \overline{\mathbb{R}}$,

$$
\mathcal{M}^{*} u(x)=\sup _{k \in \mathbb{Z}}|u|_{2^{k}}(x) .
$$

Similarly, we define median versions of a discrete convolution and a discrete maximal function. Below, balls $B_{i}$ and functions $\varphi_{i}$ are as above.

Definition 2.10. Let $0<\gamma \leq 1 / 2$. A discrete $\gamma$-median convolution of a function $u \in L^{0}(X)$ at scale $r>0$ is

$$
u_{r}^{\gamma}=\sum_{i \in I} m_{u}^{\gamma}\left(B_{i}\right) \varphi_{i}
$$

and the discrete $\gamma$-median maximal function of $u$ is $\mathcal{M}^{\gamma, *} u: X \rightarrow \overline{\mathbb{R}}$,

$$
\mathcal{M}^{\gamma, *} u(x)=\sup _{k \in \mathbb{Z}}|u|_{2^{k}}^{\gamma}(x) .
$$


Notice that taking the usual discrete convolution is a linear operation in the sense that

$$
(u+v)_{r}=u_{r}+v_{r}
$$

but the discrete median convolutions only satisfy

$$
(u+v)_{r}^{\gamma} \leq u_{r}^{\gamma / 2}+v_{r}^{\gamma / 2} .
$$

By [26, Lemma 3.1], there exists a constant $C=C\left(c_{d}\right)$ such that

$$
C^{-1} \mathcal{M} u \leq \mathcal{M}^{*} u \leq C \mathcal{M} u .
$$

Similarly, it is easy to see that there is a constant $C>0$ such that

$$
\mathcal{M}^{\gamma} u \leq C \mathcal{M}^{\gamma / C, *} u \leq C^{2} \mathcal{M}^{\gamma / C^{2}} u \text {. }
$$

As suprema of continuous functions, the discrete maximal functions are lower semicontinuous and hence measurable.

Remark 2.11. If $u \in L^{0}(X)$, then $\mathcal{M}^{\gamma} u$ may be infinite at every point. However, if $\mathcal{M}^{\gamma} u\left(x_{0}\right)<\infty$ at some point $x_{0} \in X$, then $\mathcal{M}^{\gamma} u<\infty$ almost everywhere. Indeed, at almost every point, $\lim _{r \rightarrow 0} m_{|u|}^{\gamma}(B(x, r))=|u(x)|<\infty$, which implies that there exists $r_{0}>0$ such that $m_{|u|}^{\gamma}(B(x, r)) \leq|u(x)|+1$ for $r<r_{0}$. On the other hand, if $r \geq r_{0}$, then $B(x, r) \subset B\left(x_{0}, r+d\left(x, x_{0}\right)\right)$ and there exists a constant $C=C\left(c_{d}, r_{0}, d\left(x, x_{0}\right)\right)$ such that $\mu\left(B\left(x_{0}, r+d\left(x, x_{0}\right)\right)\right) \leq C \mu(B(x, r))$, which implies that $m_{|u|}^{\gamma}(B(x, r)) \leq \mathcal{M}^{\gamma / C} u\left(x_{0}\right)$.

\section{Approximation by discrete median convolutions}

In this section, we prove Theorem 1.1 in the setting of a doubling metric space without any additional assumption on the measure. For the proof, we need a couple of lemmas including a Leibniz type rule for fractional $s$-gradients, a Sobolev-Poincaré type inequality and a pointwise estimate for $\gamma$-medians.

Lemma 3.1. Let $0<s \leq 1$ and let $S \subset X$ be a measurable set. Let $u: X \rightarrow \mathbb{R}$ be a measurable function with $\left(g_{k}\right)_{k \in \mathbb{Z}} \in \mathbb{D}^{s}(u)$ and let $\varphi$ be a bounded L-Lipschitz

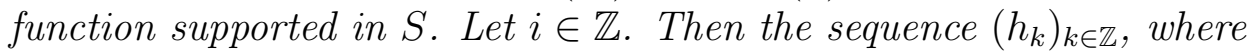

$$
h_{k}=\left\{\begin{array}{ll}
\left(\|\varphi\|_{\infty} g_{k}+L 2^{k(s-1)}|u|\right) \chi_{S}, & \text { when } k>i \\
2^{(k+1) s}\|\varphi\|_{\infty}|u| \chi_{S}, & \text { when } k \leq i
\end{array},\right.
$$

is a fractional s-gradient of function $u \varphi$.

Proof. By [20, Lemma 3.10], the sequence $\left(\hat{h}_{k}\right)_{k \in \mathbb{Z}}$, where

$$
\hat{h}_{k}=\left(\|\varphi\|_{\infty} g_{k}+L 2^{k(s-1)}|u|\right) \chi_{S}
$$

is a fractional $s$-gradient of $u \varphi$. On the other hand, by denoting

$$
\tilde{h}_{k}=2^{(k+1) s}\|\varphi\|_{\infty}|u| \chi_{S}
$$

we have

$$
\begin{aligned}
|(u \varphi)(x)-(u \varphi)(y)| & \leq\|\varphi\|_{\infty}|u(x)| \chi_{S}(x)+\|\varphi\|_{\infty}|u(y)| \chi_{S}(y) \\
& \leq d(x, y)^{s}\left(\tilde{h}_{k}(x)+\tilde{h}_{k}(y)\right),
\end{aligned}
$$


when $2^{-k-1} \leq d(x, y)<2^{-k}$, which implies that $\left(\tilde{h}_{k}\right)_{k \in \mathbb{Z}} \in \mathbb{D}^{s}(u \varphi)$. Thus, for every $i \in \mathbb{Z},\left(h_{k}\right)_{k \in \mathbb{Z}} \in \mathbb{D}^{s}(u \varphi)$.

Notice that $\tilde{h}_{k}<\hat{h}_{k}$, when $2^{-k}>2\|\varphi\|_{\infty} / L$. Hence we usually choose $i$ in (3.1) such that $2^{-i} \approx\|\varphi\|_{\infty} / L$.

The definition of fractional $s$-gradients implies the validity of various Poincaré type and pointwise inequalities. Our next lemma follows from the Sobolev-Poincaré inequality formulated in terms of integral averages in [15, Lemma 2.1] and Lemma $2.7(\mathrm{~h})$.

Lemma 3.2. Let $0<\gamma \leq 1 / 2$ and $0<s, t<\infty$. There exist constants $0<s^{\prime}<s$ and $C>0$ such that

$$
\inf _{c \in \mathbb{R}} m_{|u-c|}^{\gamma}\left(B\left(x, 2^{-k}\right)\right) \leq C 2^{-k s} \sum_{l \geq k-2} 2^{(k-l) s^{\prime}}\left(f_{B\left(x, 2^{-k+1}\right)} g_{l}^{t} d \mu\right)^{1 / t}
$$

for all $u \in L^{0}(X),\left(g_{k}\right)_{k} \in \mathbb{D}^{s}(u), x \in X$ and $k \in \mathbb{Z}$.

Notice that by Lemma 2.7 (b), (d) and (f),

$$
m_{\left|u-m_{u}^{\gamma}(A)\right|}^{\gamma}(A) \leq 2 \inf _{c \in \mathbb{R}} m_{|u-c|}^{\gamma}(A)
$$

whenever $0<\mu(A)<\infty$ and $u \in L^{0}(A)$.

Using a chaining argument and (3.2), we obtain the following pointwise estimate.

Lemma 3.3. Let $0<\gamma \leq 1 / 2$ and $0<s, t<\infty$. There exist constants $0<s^{\prime}<s$ and $C>0$ such that

$$
\left|u(x)-m_{u}^{\gamma}\left(B\left(y, 2^{-i}\right)\right)\right| \leq C 2^{-i s} \sum_{l>i-5} 2^{(i-l) s^{\prime}}\left(\mathcal{M} g_{l}^{t}(x)\right)^{1 / t}
$$

for all $u \in L^{0}(X),\left(g_{k}\right)_{k} \in \mathbb{D}^{s}(u), y \in X, i \in \mathbb{Z}$ and almost every $x \in B\left(y, 2^{-i+1}\right)$.

Proof. Let $x \in B\left(y, 2^{-i+1}\right)$ be a generalized Lebesgue point of $u$. Then, by Lemma $2.7(\mathrm{j}),(\mathrm{d}),(\mathrm{f}),(\mathrm{c}),(3.3)$ and (3.2), we obtain

$$
\begin{aligned}
\left|u(x)-m_{u}^{\gamma}\left(B\left(x, 2^{-i+2}\right)\right)\right| & \leq \sum_{k>i-3}\left|m_{u}^{\gamma}\left(B\left(x, 2^{-k-1}\right)\right)-m_{u}^{\gamma}\left(B\left(x, 2^{-k}\right)\right)\right| \\
& \leq \sum_{k>i-3} m_{\left|u-m_{u}^{\gamma}\left(B\left(x, 2^{-k}\right)\right)\right|}^{\gamma}\left(B\left(x, 2^{-k-1}\right)\right) \\
& \leq C \sum_{k>i-3} m_{\left|u-m_{u}^{\gamma}\left(B\left(x, 2^{-k}\right)\right)\right|}^{\gamma / C}\left(B\left(x, 2^{-k}\right)\right) \\
& \leq C \sum_{k>i-3} 2^{-k s} \sum_{l \geq k-2} 2^{(k-l) s^{\prime}}\left(f_{B\left(x, 2^{-k+1}\right)} g_{l}^{t} d \mu\right)^{1 / t} \\
& \leq C \sum_{l>i-5} 2^{-l s^{\prime}}\left(\mathcal{M} g_{l}^{t}(x)\right)^{1 / t} \sum_{i-3<k \leq l+2} 2^{k\left(s^{\prime}-s\right)} \\
& \leq C 2^{-i s} \sum_{l>i-5} 2^{(i-l) s^{\prime}}\left(\mathcal{M} g_{l}^{t}(x)\right)^{1 / t} .
\end{aligned}
$$


Since $B\left(y, 2^{-i}\right) \subset B\left(x, 2^{-i+2}\right)$, Lemma 2.7 (d), (f), (c), (3.3) and (3.2) imply that

$$
\begin{aligned}
\left|m_{u}^{\gamma}\left(B\left(y, 2^{-i}\right)\right)-m_{u}^{\gamma}\left(B\left(x, 2^{-i+2}\right)\right)\right| & \leq C m_{\mid u-u_{B(x, 2-i+2)}^{\gamma / C}}^{\gamma\left(B\left(x, 2^{-i+2}\right)\right)} \\
& \leq C 2^{-i s} \sum_{l>i-5} 2^{(i-l) s^{\prime}}\left(f_{B\left(x, 2^{-i+3}\right)} g_{l}^{t} d \mu\right)^{1 / t} \\
& \leq C 2^{-i s} \sum_{l>i-5} 2^{(i-l) s^{\prime}}\left(\mathcal{M} g_{l}^{t}(x)\right)^{1 / t}
\end{aligned}
$$

The claim follows by combining the above estimates.

Lemma 3.4. Let $0<p, q, \delta<\infty$ and $i \in \mathbb{Z}$.

a) If $0<t<p$, then

$$
\left\|\sum_{l>i} 2^{-(l-i) \delta}\left(\mathcal{M} g_{l}^{t}\right)^{1 / t}\right\|_{L^{p}(X)} \leq C\left(\sum_{l>i}\left\|g_{l}\right\|_{L^{p}(X)}^{q}\right)^{1 / q} .
$$

b) If $0<t<\min \{p, q\}$, then

$$
\left\|\sum_{l>i} 2^{-(l-i) \delta}\left(\mathcal{M} g_{l}^{t}\right)^{1 / t}\right\|_{L^{p}(X)} \leq C\left\|\left(\sum_{l>i} g_{l}^{q}\right)^{1 / q}\right\|_{L^{p}(X)} .
$$

Proof. a) Let $r=\min \{1, p\}$. By the Hölder inequality if $p>1$ and by the elementary inequality (2.3) if $p \leq 1$, we have

$$
\left(\sum_{l>i} 2^{-(l-i) \delta}\left(\mathcal{M} g_{l}^{t}\right)^{1 / t}\right)^{p} \leq C \sum_{l>i} 2^{-(l-i) \delta r}\left(\mathcal{M} g_{l}^{t}\right)^{p / t}
$$

Hence

$$
\begin{aligned}
\left\|\sum_{l>i} 2^{-(l-i) \delta}\left(\mathcal{M} g_{l}^{t}\right)^{1 / t}\right\|_{L^{p}(X)}^{p} & \leq \sum_{l>i} 2^{-(l-i) \delta r}\left\|\left(\mathcal{M} g_{l}^{t}\right)\right\|_{L^{p / t}(X)}^{p / t} \\
& \leq C \sum_{l>i} 2^{-(l-i) \delta r}\left\|g_{l}\right\|_{L^{p}(X)}^{p} \\
& \leq C\left(\sum_{l>i}\left\|g_{l}\right\|_{L^{p}(X)}^{q}\right)^{p / q}
\end{aligned}
$$

where the second estimate follows from the Hardy-Littlewood maximal theorem and the last one from the Hölder inequality if $q>p$ and from (2.3) if $q \leq p$.

b) By the Hölder inequality if $q>1$ and by $(2.3)$ if $q \leq 1$,

$$
\sum_{l>i} 2^{-(l-i) \delta}\left(\mathcal{M} g_{l}^{t}\right)^{1 / t} \leq C\left(\sum_{l>i}\left(\mathcal{M} g_{l}^{t}\right)^{q / t}\right)^{1 / q}
$$


Hence, by the Fefferman-Stein maximal theorem,

$$
\begin{aligned}
\left\|\sum_{l>i} 2^{-(l-i) \delta}\left(\mathcal{M} g_{l}^{t}\right)^{1 / t}\right\|_{L^{p}(X)} & \leq C\left\|\left(\sum_{l>i}\left(\mathcal{M} g_{l}^{t}\right)^{q / t}\right)^{t / q}\right\|_{L^{p / t}(X)}^{1 / t} \\
& \leq C\left\|\left(\sum_{l>i} g_{l}^{q}\right)^{t / q}\right\|_{L^{p / t}(X)}^{1 / t} \\
& =C\left\|\left(\sum_{l>i} g_{l}^{q}\right)^{1 / q}\right\|_{L^{p}(X)} .
\end{aligned}
$$

Proof of Theorem 1.1. Let $u \in \dot{N}_{p, q}^{s}(X)$ and let $u_{i}=u_{2^{-i}}^{\gamma}, i \in \mathbb{N}$. By the definition of the discrete $\gamma$-median convolution and the properties of the functions $\varphi_{j}$,

$$
u-u_{i}=\sum_{j \in J}\left(u-m_{u}^{\gamma}\left(B_{j}\right)\right) \varphi_{j}
$$

Since each $\varphi_{j}$ is $C 2^{i}$-Lipschitz and supported in $2 B_{j}$, Lemma 3.1 implies that the sequence $\left(C h_{k}^{j}\right)_{k \in \mathbb{Z}}$, where

$$
h_{k}^{j}=\left\{\begin{array}{ll}
\left(g_{k}+2^{i+k(s-1)}\left|u-m_{u}^{\gamma}\left(B_{j}\right)\right|\right) \chi_{2 B_{j}}, & \text { when } k>i \\
2^{k s}\left|u-m_{u}^{\gamma}\left(B_{j}\right)\right| \chi_{2 B_{j}}, & \text { when } k \leq i
\end{array},\right.
$$

is a fractional $s$-gradient of the function $\left(u-m_{u}^{\gamma}\left(B_{j}\right)\right) \varphi_{j}$.

Let $\left(g_{k}\right)_{k \in \mathbb{Z}} \in \mathbb{D}^{s}(u)$ and $0<t<\min \{p, q\}$. Then, by Lemma 5.3 and by the

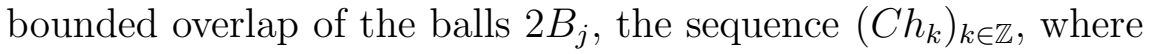

$$
h_{k}=\left\{\begin{array}{ll}
g_{k}+2^{(k-i)(s-1)} \sum_{l>i-5} 2^{(i-l) s^{\prime}}\left(\mathcal{M} g_{l}^{t}\right)^{1 / t}, & \text { when } k>i \\
2^{(k-i) s} \sum_{l>i-5} 2^{(i-l) s^{\prime}}\left(\mathcal{M} g_{l}^{t}\right)^{1 / t}, & \text { when } k \leq i
\end{array},\right.
$$

is a fractional $s$-gradient of $u-u_{i}$.

By Lemma 3.4, we have

$$
\left(\sum_{k \in \mathbb{Z}}\left\|h_{k}\right\|_{L^{p}(X)}^{q}\right)^{1 / q} \leq C\left(\sum_{l>i-5}\left\|g_{l}\right\|_{L^{p}(X)}^{q}\right)^{1 / q}
$$

and by Lemmas 3.3 and 3.4 ,

$$
\left\|u-u_{i}\right\|_{L^{p}(X)} \leq C 2^{-i s}\left(\sum_{l>i-5}\left\|g_{l}\right\|_{L^{p}(X)}^{q}\right)^{1 / q} .
$$

Thus, if $u \in \dot{N}_{p, q}^{s}(X)$, then $\left\|u-u_{i}\right\|_{N_{p, q}^{s}(X)} \rightarrow 0$ as $i \rightarrow \infty$.

Similarly, by Lemma 3.4 ,

$$
\left\|\left(\sum_{k \in \mathbb{Z}} h_{k}^{q}\right)^{1 / q}\right\|_{L^{p}(X)} \leq C\left\|\left(\sum_{l>i-5} g_{l}^{q}\right)^{1 / q}\right\|_{L^{p}(X)}
$$

and by Lemmas 3.3 and 3.4 ,

$$
\left\|u-u_{i}\right\|_{L^{p}(X)} \leq C 2^{-i s}\left\|\left(\sum_{l>i-5} g_{l}^{q}\right)^{1 / q}\right\|_{L^{p}(X)} .
$$


So, if $u \in \dot{M}_{p, q}^{s}(X)$, then $\left\|u-u_{i}\right\|_{M_{p, q}^{s}(X)} \rightarrow 0$ as $i \rightarrow \infty$.

The following example shows that discrete (median) convolution approximations of a Hajłasz function $u \in M^{1, p}(X)$ do not necessarily converge to $u$ in $M^{1, p}(X)$.

Example 3.5. Let $X=[0,1]$ and let $d$ and $\mu$ be the restrictions of the Euclidean distance and the Lebesgue measure to $[0,1]$. Let $i \in \mathbb{N}$. For $j=0,1, \ldots, 2^{i}$, let $B_{j}=B\left(j 2^{-i}, 2^{-i}\right)$ and

$$
\varphi_{j}(x)=\max \left\{0,1-2^{-i-1} d\left(x, B\left(j 2^{-i}, 2^{-i-2}\right)\right)\right\} .
$$

Then $\left\{\varphi_{j}\right\}_{j}$ is a partition of unity subordinate to the covering $\left\{B_{j}\right\}_{j}$ of $X$. If $x \in B\left(j 2^{-i}, 2^{-i-2}\right)$, then $\varphi_{j}(x)=1$ and $\varphi_{k}(x)=0$ for $k \neq j$.

Let $u: X \rightarrow \mathbb{R}, u(x)=x$. Then, for each $x \in B\left(j 2^{-i}, 2^{-i-2}\right)$,

$$
u_{2^{-i}}^{\gamma}(x)=\sum_{k} m_{u}^{\gamma}\left(B_{k}\right) \varphi_{k}(x)=m_{u}^{\gamma}\left(B_{j}\right) .
$$

Hence, if $x, y \in B\left(j 2^{-i}, 2^{-i-2}\right)$, then

$$
\left(u-u_{2^{-i}}^{\gamma}\right)(x)-\left(u-u_{2^{-i}}^{\gamma}\right)(y)=u(x)-u(y)=x-y .
$$

It follows that any $g \in \mathcal{D}^{1}\left(u-u_{2^{-i}}^{\gamma}\right)$ must satisfy $g(x) \geq 1 / 2$ for almost every $x \in \cup_{j} B\left(j 2^{-i}, 2^{-i-2}\right)$. Thus $\left\|u-u_{2^{-i}}^{\gamma}\right\|_{M^{1, p}(X)} \not \rightarrow 0$ as $i \rightarrow \infty$.

The same argument shows that the usual discrete convolutions $u_{2^{-i}}$ do not converge to $u$ in $M^{1, p}(X)$ either.

\section{Another proof of Theorem 1.1}

In this section, we give a simpler proof for Theorem 1.1 under the assumption that the underlying space $X$ has the nonempty spheres property. This proof is completely elementary and avoids the use of a chaining argument, a Sobolev-Poincaré inequality and the Fefferman-Stein maximal theorem.

Definition 4.1. A metric space $X$ has the nonempty spheres property, if there exists $R>0$ such that, for every $x \in X$ and every $0<r<R$, the set $\{y \in X: d(x, y)=r\}$ is nonempty.

Note that the nonempty spheres property implies that annuli have positive measure: Let $x \in X, 0<r<R, 0<\varepsilon<r$ and let $A=B(x, r) \backslash B(x, r-\varepsilon)$. By the assumption, there is $y$ such that $d(x, y)=r-\varepsilon / 2$. Now $B_{y}=B(y, \varepsilon / 2) \subset A$ which shows that $\mu(A) \geq \mu\left(B_{y}\right)>0$.

The nonempty spheres property allows us to prove the following estimate, which is an improved version of Lemma 3.3. Notice that, in contrast to the proof of Lemma 3.3, neither a chaining argument nor a Sobolev-Poincaré inequality is needed.

Lemma 4.2. Assume that $X$ has the nonempty spheres property. Let $0<\gamma \leq 1 / 2$ and $0<s<\infty$. Let $u \in L^{0}(X)$ and let $\left(g_{k}\right)_{k \in \mathbb{Z}} \in \mathbb{D}^{s}(u)$ be such that $g_{k} \in L^{0}(X)$ for every $k \in \mathbb{Z}$. Then there exists a constant $C=C\left(c_{d}, s\right)$ such that inequality

$$
\left|u(x)-m_{u}^{\gamma}\left(B\left(y, 2^{-i}\right)\right)\right| \leq C 2^{-i s} \mathcal{M}^{\gamma / C} \tilde{g}_{i}(x),
$$


where $\tilde{g}_{i}=\max _{i-4 \leq k \leq i} g_{k}$, holds for every $y \in X, i \in \mathbb{Z}$ with $2^{-i+3}<R$ and almost every $x \in B\left(y, 2^{-i+1}\right)$.

Proof. Denote by $E$ the set outside of which (2.2) holds. Let $x \in B\left(y, 2^{-i+1}\right) \backslash E$ be a generalized Lebesgue point of $g_{k}$ for every $k$. By Lemma $2.7(\mathrm{j})$, almost every point is such a point. Let

$$
A=B\left(y, 2^{-i+3}\right) \backslash B\left(y, 2^{-i+2}\right) .
$$

Then $A \subset B\left(x, 2^{-i+4}\right)$ and the nonempty spheres property and the doubling condition imply that $\mu\left(B\left(x, 2^{-i+4}\right)\right) \leq C \mu(A)$. By the triangle inequality, we have

$$
\left|u(x)-m_{u}^{\gamma}\left(B\left(y, 2^{-i}\right)\right)\right| \leq\left|u(x)-m_{u}^{\gamma}(A)\right|+\left|m_{u}^{\gamma}(A)-m_{u}^{\gamma}\left(B\left(y, 2^{-i}\right)\right)\right| .
$$

We begin by estimating the first term on the right-hand side of (4.1). If $z \in A \backslash E$, then $2^{-i} \leq d(x, z)<2^{-i+4}$, and hence

$$
|u(x)-u(z)| \leq 2^{(-i+4) s}\left(\tilde{g}_{i}(x)+\tilde{g}_{i}(z)\right) .
$$

Hence, using Lemma 2.7 (b)-(f), we have

$$
\begin{aligned}
\left|u(x)-m_{u}^{\gamma}(A)\right| & =\left|m_{u-u(x)}^{\gamma}(A)\right| \leq m_{|u-u(x)|}^{\gamma}(A) \\
& \leq C 2^{-i s}\left(m_{\tilde{g}_{i}}^{\gamma}(A)+\tilde{g}_{i}(x)\right) \\
& \leq C 2^{-i s}\left(m_{\tilde{g}_{i}}^{\gamma / C}\left(B\left(x, 2^{-i+4}\right)\right)+\tilde{g}_{i}(x)\right) \\
& \leq C 2^{-i s} \mathcal{M}^{\gamma / C} \tilde{g}_{i}(x) .
\end{aligned}
$$

Next, we estimate the second term on the right-hand side of (4.1). By the same argument as above,

$$
\left|u(z)-m_{u}^{\gamma}(A)\right| \leq C 2^{-i s}\left(m_{\tilde{g}_{i}}^{\gamma}(A)+\tilde{g}_{i}(z)\right)
$$

for every $z \in B\left(y, 2^{-i}\right) \backslash E$. Hence we obtain

$$
\begin{aligned}
\left|m_{u}^{\gamma}(A)-m_{u}^{\gamma}\left(B\left(y, 2^{-i}\right)\right)\right| & \leq m_{\left|u-m_{u}^{\gamma}(A)\right|}^{\gamma}\left(B\left(y, 2^{-i}\right)\right) \\
& \leq C 2^{-i s}\left(m_{\tilde{g}_{i}}^{\gamma}(A)+m_{\tilde{g}_{i}}^{\gamma}\left(B\left(y, 2^{-i}\right)\right)\right) \\
& \leq C 2^{-i s} m_{\tilde{g}_{i}}^{\gamma / C}\left(B\left(x, 2^{-i+4}\right)\right) \\
& \leq C 2^{-i s} \mathcal{M}^{\gamma / C} \tilde{g}_{i}(x),
\end{aligned}
$$

and the claim follows.

Proof of Theorem 1.1 (when $X$ satisfies the nonempty spheres property). Let $i \in \mathbb{Z}$ be such that $2^{-i+3}<R$. Let $u \in L^{0}(X)$ and $\left(g_{k}\right)_{k \in \mathbb{Z}} \in \mathbb{D}^{s}(u)$ with $g_{k} \in L^{0}(X)$ for every $k \in \mathbb{Z}$. It follows from Lemmas 3.1 and 4.2 and from the bounded overlap of the balls $2 B_{j}$ that the sequence $\left(C h_{k}\right)_{k \in \mathbb{Z}}$, where

$$
h_{k}= \begin{cases}g_{k}+2^{(k-i)(s-1)} \mathcal{M}^{\gamma / C} \tilde{g}_{i}, & \text { when } k>i \\ 2^{(k-i) s} \mathcal{M}^{\gamma / C} \tilde{g}_{i}, & \text { when } k \leq i\end{cases}
$$


and $\tilde{g}_{i}=\max _{i-4 \leq k \leq i} g_{k}$, is a fractional $s$-gradient of $u-u_{i}$. By the boundedness of $\mathcal{M}^{\gamma / C}$ in $L^{p}$, (2.7), we have

$$
\begin{aligned}
\left\|u-u_{i}\right\|_{\dot{N}_{p}^{s}, q}(X) & \leq C\left(\sum_{k \in \mathbb{Z}}\left\|h_{k}\right\|_{L^{p}(X)}^{q}\right)^{1 / q} \\
& \leq C\left(\sum_{k>i}\left\|g_{k}\right\|_{L^{p}(X)}^{q}\right)^{1 / q}+C\left\|\mathcal{M}^{\gamma / C} \tilde{g}_{i}\right\|_{L^{p}(X)} \\
& \leq C\left(\sum_{k>i-5}\left\|g_{k}\right\|_{L^{p}(X)}^{q}\right)^{1 / q}
\end{aligned}
$$

and

$$
\begin{aligned}
\left\|u-u_{i}\right\|_{\dot{M}_{p, q}^{s}(X)} & \leq C\left\|\left(\sum_{k \in \mathbb{Z}} h_{k}^{q}\right)^{1 / q}\right\|_{L^{p}(X)} \\
& \leq C\left\|\left(\sum_{k>i} g_{k}^{q}\right)^{1 / q}\right\|_{L^{p}(X)}+C\left\|\mathcal{M}^{\gamma / C} \tilde{g}_{i}\right\|_{L^{p}(X)} \\
& \leq C\left\|\left(\sum_{k>i-5} g_{k}^{q}\right)^{1 / q}\right\|_{L^{p}(X)}
\end{aligned}
$$

Moreover, by Lemma 4.2 and (2.7),

$$
\left\|u-u_{i}\right\|_{L^{p}(X)} \leq C 2^{-i s}\left\|\mathcal{M}^{\gamma / C} \tilde{g}_{i}\right\|_{L^{p}(X)} \leq C 2^{-i s}\left\|\tilde{g}_{i}\right\|_{L^{p}(X)} .
$$

The claim follows from these estimates.

\section{Approximation by discrete COnvolutions}

The main result of this section is Theorem 5.1, which is a counterpart of Theorem 1.1 for usual discrete convolutions. It shows that discrete convolutions converge to the locally integrable function in Hajłasz-Besov and Hajłasz-Triebel-Lizorkin norm if $Q /(Q+s)<p<\infty$ in the Besov case and $Q /(Q+s)<q<\infty$ in the TriebelLizorkin case. Recall from (2.9) that the discrete convolution of a locally integrable function $u$ at the scale $r$ is $u_{r}=\sum_{i \in I} u_{B_{i}} \varphi_{i}$.

Theorem 5.1. Let $0<s<1$ and $Q /(Q+s)<p<\infty$.

a) If $0<q<\infty$, then

$$
\lim _{i \rightarrow \infty}\left\|u_{2^{-i}}-u\right\|_{N_{p, q}^{s}(X)}=0
$$

for every $u \in \dot{N}_{p, q}^{s}(X)$.

b) If $Q /(Q+s)<q<\infty$, then

$$
\lim _{i \rightarrow \infty}\left\|u_{2^{-i}}-u\right\|_{M_{p, q}^{s}(X)}=0
$$

for every $u \in \dot{M}_{p, q}^{s}(X)$.

The proof is almost the same as the proof of Theorem 1.1 given in Section 3. Instead of Lemma 3.2 we use Lemma 5.2 which follows from [15, Lemma 2.1]. 
Lemma 5.2. Let $0<s<\infty$ and $t>Q /(Q+s)$. Then there exist constants $0<s^{\prime}<s$ and $C>0$ such that

$$
f_{B\left(x, 2^{-k}\right)}\left|u-u_{B\left(x, 2^{-k}\right)}\right| d \mu \leq C 2^{-k s} \sum_{l \geq k-2} 2^{(k-l) s^{\prime}}\left(f_{B\left(x, 2^{-k+1}\right)} g_{l}^{t} d \mu\right)^{1 / t}
$$

for all locally integrable functions $u$, all $\left(g_{k}\right)_{k \in \mathbb{Z}} \in \mathbb{D}^{s}(u), x \in X$ and $k \in \mathbb{Z}$. In particular, if $p>Q /(Q+s)$, then (5.1) holds for every $u \in N_{p, q}^{s}(X) \cup M_{p, q}^{s}(X)$.

A standard chaining argument and (5.1) imply the following pointwise estimate.

Lemma 5.3. Let $0<s<\infty$ and $Q /(Q+s)<t<\infty$. Then there exist constants $C>0$ and $0<s^{\prime}<s$ such that

$$
\left|u(x)-u_{B\left(y, 2^{-i}\right)}\right| \leq C 2^{-i s} \sum_{l>i-5} 2^{(i-l) s^{\prime}}\left(\mathcal{M} g_{l}^{t}(x)\right)^{1 / t}
$$

for all locally integrable functions $u$, all $\left(g_{k}\right)_{k \in \mathbb{Z}} \in \mathbb{D}^{s}(u), y \in X, i \in \mathbb{Z}$ and almost every $x \in B\left(y, 2^{-i+1}\right)$.

Proof of Theorem 5.1. a) Let $u \in \dot{N}_{p, q}^{s}(X),\left(g_{k}\right)_{k \in \mathbb{Z}} \in \mathbb{D}^{s}(u) \cap l^{q}\left(L^{p}(X)\right)$ and let $Q /(Q+s)<t<p$. Since

$$
u-u_{2^{-i}}=\sum_{j \in J}\left(u-u_{B_{j}}\right) \varphi_{j},
$$

it follows from Lemmas $3.1,5.3$ and from the bounded overlap of the balls $2 B_{j}$ that the sequence $\left(C h_{k}\right)_{k \in \mathbb{Z}}$, where

$$
h_{k}=\left\{\begin{array}{ll}
g_{k}+2^{(k-i)(s-1)} \sum_{l>i-5} 2^{(i-l) s^{\prime}}\left(\mathcal{M} g_{l}^{t}\right)^{1 / t}, & \text { when } k>i \\
2^{(k-i) s} \sum_{l>i-5} 2^{(i-l) s^{\prime}}\left(\mathcal{M} g_{l}^{t}\right)^{1 / t}, & \text { when } k \leq i
\end{array},\right.
$$

is a fractional $s$-gradient of our function $u-u_{2^{-i}}$. By Lemma 3.4, we have

$$
\left(\sum_{k \in \mathbb{Z}}\left\|h_{k}\right\|_{L^{p}(X)}^{q}\right)^{1 / q} \leq C\left(\sum_{l>i-5}\left\|g_{l}\right\|_{L^{p}(X)}^{q}\right)^{1 / q}
$$

and by Lemmas 5.3 and 3.4 ,

$$
\left\|u-u_{2^{-i}}\right\|_{L^{p}(X)} \leq C 2^{-i s}\left(\sum_{l>i-5}\left\|g_{l}\right\|_{L^{p}(X)}^{q}\right)^{1 / q} .
$$

Thus, $\left\|u-u_{2^{-i}}\right\|_{N_{p, q}^{s}(X)} \rightarrow 0$ as $i \rightarrow \infty$.

b) Let $u \in \dot{M}_{p, q}^{s}(X),\left(g_{k}\right)_{k \in \mathbb{Z}} \in \mathbb{D}^{s}(u) \cap L^{p}\left(X, l^{q}\right)$ and $Q /(Q+s)<t<\min \{p, q\}$. Then the sequence $\left(C h_{k}\right)_{k \in \mathbb{Z}}$, where $h_{k}$ is as above, is a fractional s-gradient of $u-u_{2^{-i}}$. By Lemma 3.4, we have

$$
\left\|\left(\sum_{k \in \mathbb{Z}} h_{k}^{q}\right)^{1 / q}\right\|_{L^{p}(X)} \leq C\left\|\left(\sum_{l>i-5} g_{l}^{q}\right)^{1 / q}\right\|_{L^{p}(X)}
$$


and by Lemmas 5.3 and 3.4 ,

$$
\left\|u-u_{2^{-i}}\right\|_{L^{p}(X)} \leq C 2^{-i s}\left\|\left(\sum_{l>i-5} g_{l}^{q}\right)^{1 / q}\right\|_{L^{p}(X)} .
$$

Hence $\left\|u-u_{2^{-i}}\right\|_{M_{p, q}^{s}(X)} \rightarrow 0$ as $i \rightarrow \infty$.

\section{CAPACity}

In this section, we define Hajłasz-Besov and Hajłasz-Triebel-Lizorkin capacities and prove some of their basic properties. In the Euclidean setting, Besov and Triebel-Lizorkin capacities are studied and used for example in [1, [2], 3], 4], [5], [10], [19], [34], 35], [36], [42] and in metric spaces in [7], [9] (with less than three indices in the space).

Definition 6.1. Let $0<s<\infty, 0<p, q \leq \infty$ and $\mathcal{F} \in\left\{N_{p, q}^{s}, M_{p, q}^{s}\right\}$. The $\mathcal{F}$ capacity of a set $E \subset X$ is

$$
C_{\mathcal{F}}(E)=\inf \left\{\|u\|_{\mathcal{F}}^{p}: u \in \mathcal{A}_{\mathcal{F}}(E)\right\}
$$

where

$$
\mathcal{A}_{\mathcal{F}}(E)=\{u \in \mathcal{F}: u \geq 1 \text { on a neighbourhood of } E\}
$$

is a set of admissible functions for the capacity. We say that a property holds $\mathcal{F}$-quasieverywhere if it holds outside a set of $\mathcal{F}$-capacity zero.

Remark 6.2. Lemma 2.3 easily implies that

$$
C_{\mathcal{F}}(E)=\inf \left\{\|u\|_{\mathcal{F}}^{p}: u \in \mathcal{A}_{\mathcal{F}}^{\prime}(E)\right\}
$$

where $\mathcal{A}_{\mathcal{F}}^{\prime}(E)=\left\{u \in \mathcal{A}_{\mathcal{F}}(E): 0 \leq u \leq 1\right\}$.

Remark 6.3. It is easy to see that the $\mathcal{F}$-capacity is an outer capacity, which means that

$$
C_{\mathcal{F}}(E)=\inf \left\{C_{\mathcal{F}}(U): U \supset E, U \text { is open }\right\} .
$$

The $\mathcal{F}$-capacity is not generally subadditive, but for most purposes, it suffices that it satisfies (6.1) for some $r>0$. Even in the classical case, countable subadditivity for Besov-capacity is known only when $p \leq q$, see [1].

Lemma 6.4. Let $0<s<\infty, 0<p \leq \infty, 0<p \leq \infty$ and let $\mathcal{F} \in\left\{N_{p, q}^{s}, M_{p, q}^{s}\right\}$. Then there are constants $c \geq 1$ and $0<r \leq 1$ such that

$$
C_{\mathcal{F}}\left(\bigcup_{i \in \mathbb{N}} E_{i}\right)^{r} \leq c \sum_{i \in \mathbb{N}} C_{\mathcal{F}}\left(E_{i}\right)^{r}
$$

for all sets $E_{i} \subset X, i \in \mathbb{N}$. Actually, (6.1) holds with $r=\min \{1, q / p\}$.

Proof. Let $r=\min \{1, q / p\}$ and $E=\cup_{i \in \mathbb{N}} E_{i}$. Let $\varepsilon>0$.

We prove the case $\mathcal{F}=N_{p, q}^{s}$ first. We may assume that $\sum_{i \in \mathbb{N}} C_{N_{p, q}^{s}}\left(E_{i}\right)^{r}<\infty$. There are functions $u_{i} \in \mathcal{A}_{N_{p, q}^{s}}^{\prime}\left(E_{i}\right)$ with $\left(g_{i, k}\right)_{k \in \mathbb{Z}} \in \mathbb{D}^{s}\left(u_{i}\right)$ such that

$$
\left(\left\|u_{i}\right\|_{L^{p}(X)}+\left\|\left(g_{i, k}\right)_{k}\right\|_{l^{q}\left(L^{p}(X)\right)}\right)^{p r}<C_{N_{p, q}^{s}}\left(E_{i}\right)^{r}+2^{-i} \varepsilon .
$$


Then $u=\sup _{i \in \mathbb{N}} u_{i} \in \mathcal{A}_{N_{p, q}^{s}}^{\prime}(E)$ and the sequence $\left(g_{k}\right)_{k \in \mathbb{Z}}=\left(\sup _{i \in \mathbb{N}} g_{i, k}\right)_{k \in \mathbb{Z}}$ is a fractional $s$-gradient of $u$. We have $\left\|g_{k}\right\|_{L^{p}(X)}^{p} \leq \sum_{i \in \mathbb{N}}\left\|g_{i, k}\right\|_{L^{p}(X)}^{p}$, for every $k$, and so

$$
\begin{aligned}
\left\|\left(g_{k}\right)_{k}\right\|_{l^{q}\left(L^{p}(X)\right)}^{p r} & =\left\|\left(\left\|g_{k}\right\|_{L^{p}(X)}^{p}\right)_{k}\right\|_{l^{q} / p}^{r} \leq\left\|\sum_{i \in \mathbb{N}}\left(\left\|g_{i, k}\right\|_{L^{p}(X)}^{p}\right)_{k}\right\|_{l^{q / p}}^{r} \\
& \leq \sum_{i \in \mathbb{N}}\left\|\left(\left\|g_{i, k}\right\|_{L^{p}(X)}^{p}\right)_{k}\right\|_{l^{q / p}}^{r}=\sum_{i \in \mathbb{N}}\left\|\left(g_{i, k}\right)_{k}\right\|_{l^{q}\left(L^{p}(X)\right)}^{p r}
\end{aligned}
$$

Here we also used the fact that

$$
\left\|\sum_{k \in \mathbb{N}}\left(a_{i}^{k}\right)_{i \in \mathbb{Z}}\right\|_{l^{q / p}}^{r} \leq \sum_{k \in \mathbb{N}}\left\|\left(a_{i}^{k}\right)_{i \in \mathbb{Z}}\right\|_{l^{q / p}}^{r}
$$

for all $\left(a_{i}^{k}\right)_{i \in \mathbb{Z}} \in l^{q / p}, k \in \mathbb{N}$. Since $\|u\|_{L^{p}(X)}^{p r} \leq \sum_{i \in \mathbb{N}}\left\|u_{i}\right\|_{L^{p}(X)}^{p r}$, we have that

$$
\begin{aligned}
C_{N_{p, q}^{s}}(E)^{r} & \leq 2^{p r}\left(\|u\|_{L^{p}(X)}^{p r}+\left\|\left(g_{k}\right)_{k}\right\|_{l^{q}\left(L^{p}(X)\right)}^{p r}\right) \\
& \leq 2^{p r} \sum_{i \in \mathbb{N}}\left(\left\|u_{i}\right\|_{L^{p}(X)}^{p r}+\left\|\left(g_{i, k}\right)_{k}\right\|_{l^{q}\left(L^{p}(X)\right)}^{p r}\right) \\
& \leq 2^{p r+1} \sum_{i \in \mathbb{N}}\left(\left\|u_{i}\right\|_{L^{p}(X)}+\left\|\left(g_{i, k}\right)_{k}\right\|_{l^{q}\left(L^{p}(X)\right)}\right)^{p r} \\
& \leq 2^{p r+1}\left(\sum_{i \in \mathbb{N}} C_{N_{p, q}^{s}}\left(E_{i}\right)^{r}+\varepsilon\right) .
\end{aligned}
$$

The claim follows by letting $\varepsilon \rightarrow 0$.

Assume then that $\sum_{i \in \mathbb{N}} C_{M_{p, q}^{s}}\left(E_{i}\right)^{r}<\infty$. Let $u_{i} \in \mathcal{A}_{M_{p, q}^{s}}^{\prime}\left(E_{i}\right)$ and $\left(g_{i, k}\right)_{k \in \mathbb{Z}} \in$ $\mathbb{D}^{s}\left(u_{i}\right)$ be such that

$$
\left(\left\|u_{i}\right\|_{L^{p}(X)}+\left\|\left(g_{i, k}\right)_{k}\right\|_{L^{p}\left(X, l^{q}\right)}\right)^{p r}<C_{M_{p, q}^{s}}\left(E_{i}\right)^{r}+2^{-i} \varepsilon
$$

Then $u=\sup _{i \in \mathbb{N}} u_{i}$ belongs to $\mathcal{A}_{M_{p, q}^{s}}^{\prime}(E)$ and $\left(g_{k}\right)_{k \in \mathbb{Z}}=\left(\sup _{i \in \mathbb{N}} g_{i, k}\right)_{k \in \mathbb{Z}}$ is a fractional $s$-gradient of $u$. Since $g_{k}^{q} \leq \sum_{i} g_{i, k}^{q}$, for every $k$, it follows that

$$
\begin{aligned}
\left\|\left(g_{k}\right)_{k}\right\|_{L^{p}\left(X, l^{q}\right)}^{p r} & =\left\|\left(\sum_{k \in \mathbb{Z}} g_{k}^{q}\right)^{1 / q}\right\|_{L^{p}(X)}^{p r} \leq\left\|\sum_{k \in \mathbb{Z}} \sum_{i \in \mathbb{N}} g_{i, k}^{q}\right\|_{L^{p / q}(X)}^{p r / q} \\
& =\left\|\sum_{i \in \mathbb{N}} \sum_{k \in \mathbb{Z}} g_{i, k}^{q}\right\|_{L^{p / q}(X)}^{\min \{1, p / q\}} \leq \sum_{i \in \mathbb{N}}\left\|\sum_{k \in \mathbb{Z}} g_{i, k}^{q}\right\|_{L^{p / q}(X)}^{\min \{1, p / q\}} \\
& =\sum_{i \in \mathbb{N}}\left\|\left(\sum_{k \in \mathbb{Z}} g_{i, k}^{q}\right)^{1 / q}\right\|_{L^{p}(X)}^{p r}=\sum_{i \in \mathbb{N}}\left\|\left(g_{i, k}\right)_{k}\right\|_{L^{p}\left(X, l^{q}\right)}^{p r} .
\end{aligned}
$$

The rest of the proof is the same as in the Besov case.

\section{Quasicontinuity and Generalized Lebesgue Points}

In this section, we prove the second main theorem of the paper, Theorem 1.2 , which shows that quasievery point is a generalized Lebesgue points of any given Hajłasz-Besov or Hajłasz-Triebel-Lizorkin function and that the limit of medians gives a quasicontinuous representative of the function. 
As usual, we say that a function $u$ is $\mathcal{F}$-quasicontinuous, if for every $\varepsilon>0$, there exists a set $U$ such that $C_{\mathcal{F}}(U)<\varepsilon$ and the restriction of $u$ to $X \backslash U$ is continuous. By Remark 6.3, the set $U$ can be chosen to be open.

The main ingredient of the proof of Theorem 1.2 is a capacitary weak-type estimate for the median maximal function proved in Theorem 7.7 below. This estimate follows immediately from the fact that the discrete median maximal operator is bounded on $\mathcal{F}(X)$ (Theorem 7.6). We begin with a lemma, which gives a formula for a fractional $s$-gradient of the discrete median maximal function.

Lemma 7.1. Let $0<\gamma \leq 1 / 2,0<s<1$ and $0<t<\infty$. Let $u \in L^{0}(X)$ and $\left(g_{k}\right)_{k \in \mathbb{Z}} \in \mathbb{D}^{s}(u)$. Then there exist constants $C>0$ and $0<s^{\prime}<s$ such that $\left(C \tilde{g}_{k}\right)_{k \in \mathbb{Z}}$, where

$$
\tilde{g}_{k}=\sum_{l \in \mathbb{Z}} 2^{-|l-k| \delta}\left(\mathcal{M} g_{l}^{t}\right)^{1 / t}
$$

and $\delta=\min \left\{s^{\prime}, 1-s\right\}$, is a fractional s-gradient of the discrete $\gamma$-median convolution approximation $u_{2^{-i}}^{\gamma}$ for every $i \in \mathbb{Z}$. Consequently, if $\mathcal{M}^{\gamma, *} u \not \equiv \infty$, then $\left(C \tilde{g}_{k}\right)_{k \in \mathbb{Z}}$ is a fractional s-gradient of $\mathcal{M}^{\gamma, *} u$.

Proof. By the proof of Theorem 1.1, the sequence $\left(C h_{k}\right)_{k \in \mathbb{Z}}$, where

$$
h_{k}=\left\{\begin{array}{ll}
g_{k}+2^{(i-k)(1-s)} \sum_{l>i-5} 2^{(i-l) s^{\prime}}\left(\mathcal{M} g_{l}^{t}\right)^{1 / t}, & \text { when } k>i \\
2^{(k-i) s} \sum_{l>i-5} 2^{(i-l) s^{\prime}}\left(\mathcal{M} g_{l}^{t}\right)^{1 / t}, & \text { when } k \leq i
\end{array},\right.
$$

is a fractional $s$-gradient of our function $u-u_{2^{-i}}^{\gamma}$. It follows that $\left(C \tilde{h}_{k}\right)_{k \in \mathbb{Z}}$, where

$$
\tilde{h}_{k}=\left\{\begin{array}{ll}
g_{k}+2^{(i-k)(1-s)} \sum_{l>i-5} 2^{(i-l) s^{\prime}}\left(\mathcal{M} g_{l}^{t}\right)^{1 / t}, & \text { when } k>i \\
g_{k}+2^{(k-i) s} \sum_{l>i-5} 2^{(i-l) s^{\prime}}\left(\mathcal{M} g_{l}^{t}\right)^{1 / t}, & \text { when } k \leq i
\end{array},\right.
$$

is a fractional $s$-gradient of $u_{2^{-i}}^{\gamma}$. Clearly, $g_{k} \leq \tilde{g}_{k}$ almost everywhere for every $k$.

If $k>i$, then

$$
2^{(i-k)(1-s)} \sum_{i-5<l<k} 2^{(i-l) s^{\prime}}\left(\mathcal{M} g_{l}^{t}\right)^{1 / t} \leq C \sum_{i-5<l<k} 2^{(l-k)(1-s)}\left(\mathcal{M} g_{l}^{t}\right)^{1 / t}
$$

and

$$
2^{(i-k)(1-s)} \sum_{l \geq k} 2^{(i-l) s^{\prime}}\left(\mathcal{M} g_{l}^{t}\right)^{1 / t} \leq \sum_{l \geq k} 2^{(k-l) s^{\prime}}\left(\mathcal{M} g_{l}^{t}\right)^{1 / t}
$$

If $k \leq i$, then

$$
\begin{aligned}
2^{(k-i) s} \sum_{l>i-5} 2^{(i-l) s^{\prime}}\left(\mathcal{M} g_{l}^{t}\right)^{1 / t} & =2^{(k-i)\left(s-s^{\prime}\right)} \sum_{l>i-5} 2^{(k-l) s^{\prime}}\left(\mathcal{M} g_{l}^{t}\right)^{1 / t} \\
& \leq \sum_{l>k-5} 2^{(k-l) s^{\prime}}\left(\mathcal{M} g_{l}^{t}\right)^{1 / t}
\end{aligned}
$$

Thus, $\tilde{h}_{k} \leq C \tilde{g}_{k}$ almost everywhere, for every $k \in \mathbb{Z}$, and so $\left(\tilde{g}_{k}\right)_{k \in \mathbb{Z}} \in \mathbb{D}^{s}\left(u_{2^{-i}}^{\gamma}\right)$.

If $\mathcal{M}^{\gamma, *} u \not \equiv \infty$, then by Remark 2.11, $\mathcal{M}^{\gamma, *} u \in L^{0}(X)$. Hence, by Lemma 2.4, $\left(C \tilde{g}_{k}\right)_{k \in \mathbb{Z}} \in \mathbb{D}^{s}\left(\mathcal{M}^{\gamma, *} u\right)$. 
From the proof of the above lemma, we see that if $g$ is an $s$-gradient of $u$ and $0<t<\infty$, then there exists a constant $C>0$ such that $C\left(\mathcal{M} g^{t}\right)^{1 / t}$ is an $s$-gradient of $u_{2^{-i}}^{\gamma}$ for every $i$. Below, we show that an even better result holds. We need the following lemma, which is a special case of Lemma 3.1.

Lemma 7.2. Let $0<s \leq 1$ and let $S \subset X$ be a measurable set. Let $u: X \rightarrow \mathbb{R}$ be a measurable function with $g \in \mathcal{D}^{s}(u)$ and let $\varphi$ be a bounded L-Lipschitz function supported in $S$. Then

$$
h=\left(\|\varphi\|_{\infty} g+\left(2\|\varphi\|_{\infty}\right)^{1-s} L^{s}|u|\right) \chi_{S} \in \mathcal{D}^{s}(u \varphi) .
$$

Lemma 7.3. Let $0<s \leq 1$ and $0<\gamma \leq 1 / 2$. Let $u \in L^{0}(X)$ and $g \in \mathcal{D}^{s}(u) \cap$ $L^{0}(X)$. Then there exists a constant $C>0$ such that $C \mathcal{M}^{\gamma / C} g$ is an s-gradient of $u_{r}^{\gamma}$ for every $r>0$. Consequently, if $\mathcal{M}^{\gamma, *} u \not \equiv \infty$, then $C \mathcal{M}^{\gamma / C} g$ is an s-gradient of $\mathcal{M}^{\gamma, *} u$.

Proof. By the definition of the discrete $\gamma$-median convolution and the properties of the functions $\varphi_{i}$,

$$
u_{r}^{\gamma}=u+\sum_{i \in \mathbb{N}}\left(m_{u}^{\gamma}\left(B_{i}\right)-u\right) \varphi_{i}
$$

and by Lemma $\mathbf{7 . 2}$, the function

$$
\left(g+C r^{-s}\left|u-m_{u}^{\gamma}\left(B_{i}\right)\right|\right) \chi_{2 B_{i}}
$$

is an $s$-gradient of our function $\left(m_{u}^{\gamma}\left(B_{i}\right)-u\right) \varphi_{i}$ for each $i$.

Let $x \in 2 B_{i} \backslash E$, where $E$ is the exceptional set for (2.1). Using Lemma 2.7, we obtain

$$
\begin{aligned}
\left|u(x)-m_{u}^{\gamma}\left(B_{i}\right)\right| & \leq m_{|u-u(x)|}^{\gamma}\left(B_{i}\right) \leq C r^{s}\left(m_{g}^{\gamma / C}\left(B_{i}\right)+g(x)\right) \\
& \leq C r^{s}\left(m_{g}^{\gamma / C}(B(x, 3 r))+g(x)\right) \\
& \leq C r^{s}\left(g(x)+\mathcal{M}^{\gamma / C} g(x)\right) .
\end{aligned}
$$

Since $g(x) \leq \mathcal{M}^{\gamma / C} g(x)$ for almost every $x$ and since the balls $2 B_{i}$ have bounded overlap, it follows that

$$
C \mathcal{M}^{\gamma / C} g \in \mathcal{D}^{s}\left(u_{r}^{\gamma}\right)
$$

for every $r>0$.

Assume then that $\mathcal{M}^{\gamma, *} u \not \equiv \infty$. Then, by Remark 2.11, $\mathcal{M}^{\gamma, *} u \in L^{0}(X)$, and so, by Lemma 2.4 ,

$$
C \mathcal{M}^{\gamma / C} g \in \mathcal{D}^{s}\left(\mathcal{M}^{\gamma, *} u\right),
$$

from which the second claim follows.

Remark 7.4. If $X$ has the nonempty spheres property (Definition4.1) with $R=\infty$, then the proof of Theorem 1.1 given in Section 4 shows that, for $0<\gamma \leq 1 / 2$ and $0<s \leq 1$, there exists a constant $C>0$ such that $\left(C \tilde{g}_{k}\right)_{k \in \mathbb{Z}}$, where

$$
\tilde{g}_{k}=\sup _{l \in \mathbb{Z}} 2^{-|l-k| \delta} \mathcal{M}^{\gamma / C} g_{l}
$$

and $\delta=\min \{s, 1-s\}$, is a fractional $s$-gradient of $\mathcal{M}^{\gamma, *} u$, whenever $u \in L^{0}(X)$, $\left(g_{k}\right)_{k \in \mathbb{Z}} \in \mathbb{D}^{s}(u)$ and $\mathcal{M}^{\gamma, *} u \not \equiv \infty$. 
The proof of the following lemma is essentially contained in the proofs of Theorems 4.5 and 4.6 in [21], but for the convenience of the reader, we repeat the proof.

Lemma 7.5. Let $0<p, q, \delta<\infty$ and $0<t<\min \{p, q\}$. Let $\left(g_{k}\right)_{k \in \mathbb{Z}} \in l^{q}\left(L^{p}(X)\right) \cup$ $L^{p}\left(X, l^{q}\right)$ and let

$$
h_{k}=\sum_{l \in \mathbb{Z}} 2^{-|l-k| \delta}\left(\mathcal{M} g_{l}^{t}\right)^{1 / t}
$$

for every $k \in \mathbb{Z}$. Then there is a constant $C=C\left(p, q, t, \delta, c_{d}\right)$ such that

$$
\left\|\left(h_{k}\right)_{k \in \mathbb{Z}}\right\|_{l^{q}\left(L^{p}(X)\right)} \leq C\left\|\left(g_{k}\right)_{k \in \mathbb{Z}}\right\|_{l^{q}\left(L^{p}(X)\right)},
$$

if $\left(g_{k}\right)_{k \in \mathbb{Z}} \in l^{q}\left(L^{p}(X)\right)$, and

$$
\left\|\left(h_{k}\right)_{k \in \mathbb{Z}}\right\|_{L^{p}\left(X, l^{q}\right)} \leq C\left\|\left(g_{k}\right)_{k \in \mathbb{Z}}\right\|_{L^{p}\left(X, l^{q}\right)},
$$

if $\left(g_{k}\right)_{k \in \mathbb{Z}} \in L^{p}\left(X, l^{q}\right)$.

Proof. Let $\tilde{p}=\min \{1, p\}$. Using (2.4) and the Hardy-Littlewood maximal theorem, we have

$$
\left\|h_{k}\right\|_{L^{p}(X)}^{\tilde{p}} \leq \sum_{l \in \mathbb{Z}} 2^{-|l-k| \delta \tilde{p}}\left\|\left(\mathcal{M} g_{l}^{t}\right)^{1 / t}\right\|_{L^{p}(X)}^{\tilde{p}} \leq C \sum_{l \in \mathbb{Z}} 2^{-|l-k| \delta \tilde{p}}\left\|g_{l}\right\|_{L^{p}(X)}^{\tilde{p}} .
$$

Hence, by Lemma 2.6,

$$
\left\|\left(h_{k}\right)\right\|_{l^{q}\left(L^{p}(X)\right)}^{q} \leq C \sum_{k \in \mathbb{Z}}\left(\sum_{l \in \mathbb{Z}} 2^{-|l-k| \delta \tilde{p}}\left\|g_{l}\right\|_{L^{p}(X)}^{\tilde{p}}\right)^{q / \tilde{p}} \leq C \sum_{l \in \mathbb{Z}}\left\|g_{l}\right\|_{L^{p}(X)}^{q}
$$

and the first claim follows.

Since

$$
\left\|\left(h_{k}\right)\right\|_{l^{q}}^{q}=\sum_{k \in \mathbb{Z}}\left(\sum_{l \in \mathbb{Z}} 2^{-|l-k| \delta}\left(\mathcal{M} g_{l}^{t}\right)^{1 / t}\right)^{q} \leq C \sum_{k \in \mathbb{Z}}\left(\mathcal{M} g_{k}^{t}\right)^{q / t}
$$

by Lemma 2.6, the Fefferman-Stein maximal theorem implies that

$$
\left\|\left(h_{k}\right)\right\|_{L^{p}\left(X ; l^{q}\right)} \leq C\left\|\left(M g_{k}^{t}\right)\right\|_{L^{p / t}\left(X ; l^{q / t}\right)}^{1 / t} \leq C\left\|\left(g_{k}^{t}\right)_{k \in \mathbb{Z}}\right\|_{L^{p / t}\left(X ; l^{q / t}\right)}^{1 / t},=C\left\|\left(g_{k}\right)\right\|_{L^{p}\left(X, l^{q}\right)},
$$

which proves the second claim.

Next we show that the discrete median maximal operator is bounded on $\mathcal{F}(X)$ and use this result to prove a capacitary weak-type estimate for the median maximal function.

Theorem 7.6. Let $0<\gamma \leq 1 / 2$. Let $0<s<1,0<p<\infty, 0<q<\infty$ and $\mathcal{F} \in\left\{N_{p, q}^{s}, M_{p, q}^{s}\right\}$ or $0<s \leq 1,0<p<\infty$ and $\mathcal{F}=M_{p, \infty}^{s}=M^{s, p}$. Then there exists a constant $C=C\left(c_{d}, s, p, q, \gamma\right)$ such that

$$
\left\|\mathcal{M}^{\gamma, *} u\right\|_{\mathcal{F}(X)} \leq C\|u\|_{\mathcal{F}(X)}
$$

for all $u \in \mathcal{F}(X)$. 
Proof. By the boundedness of the discrete median maximal operator on $L^{p}$, which follows from (2.10) and (2.7), we have

$$
\left\|\mathcal{M}^{\gamma, *} u\right\|_{L^{p}(X)} \leq C\|u\|_{L^{p}(X)}
$$

To estimate the $s$-gradient, suppose first that $0<s<1,0<p, q<\infty, u \in N_{p, q}^{s}(X)$ and $\left(g_{k}\right)_{k \in \mathbb{Z}} \in \mathbb{D}^{s}(u)$. Let $0<t<\min \{p, q\}$. By Lemma 7.1, the sequence $\left(C \tilde{g}_{k}\right)_{k \in \mathbb{Z}}$, where

$$
\tilde{g}_{k}=\sum_{l \in \mathbb{Z}} 2^{-|l-k| \delta}\left(\mathcal{M} g_{l}^{t}\right)^{1 / t}
$$

and $\delta=\min \left\{s^{\prime}, 1-s\right\}$, is a fractional $s$-gradient of $\mathcal{M}^{\gamma, *} u$ and by Lemma 7.5 ,

$$
\left\|\left(\tilde{g}_{k}\right)_{k \in \mathbb{Z}}\right\|_{l^{q}\left(L^{p}(X)\right)} \leq C\left\|\left(g_{k}\right)_{k \in \mathbb{Z}}\right\|_{l^{q}\left(L^{p}(X)\right)} .
$$

Thus, $\left\|\mathcal{M}^{\gamma, *} u\right\|_{\dot{N}_{p, q}^{s}(X)} \leq C\|u\|_{\dot{N}_{p, q}^{s}(X)}$.

The proof for the estimate $\left\|\mathcal{M}^{\gamma, *} u\right\|_{\dot{M}_{p, q}^{s}(X)} \leq C\|u\|_{\dot{M}_{p, q}^{s}(X)}$ is analogous. Finally, the estimate $\left\|\mathcal{M}^{\gamma, *} u\right\|_{\dot{M}^{s, p}(X)} \leq C\|u\|_{\dot{M}^{s, p}(X)}$ follows from Lemma 7.3 and (2.7).

Theorem 7.7. Let $0<\gamma \leq 1 / 2$. Let $0<s<1,0<p<\infty, 0<q<\infty$ and $\mathcal{F} \in\left\{N_{p, q}^{s}, M_{p, q}^{s}\right\}$ or $0<s \leq 1,0<p<\infty$ and $\mathcal{F}=M_{p, \infty}^{s}=M^{s, p}$. Then there exists a constant $C=C\left(c_{d}, s, p, q, \gamma\right)$ such that

$$
C_{\mathcal{F}}\left(\left\{x \in X: \mathcal{M}^{\gamma} u(x)>\lambda\right\}\right) \leq C \lambda^{-p}\|u\|_{\mathcal{F}(X)}^{p}
$$

for all $u \in \mathcal{F}(X)$ and for all $\lambda>0$.

Proof. By (2.10), $\mathcal{M}^{\gamma} u \leq C \mathcal{M}^{\gamma / C, *} u$. Hence, Theorem 7.6 together with the lower semicontinuity of $\mathcal{M}^{\gamma / C, *} u$ implies that

$$
\begin{aligned}
C_{\mathcal{F}}\left(\left\{x \in X: \mathcal{M}^{\gamma} u(x)>\lambda\right\}\right) & \leq C_{\mathcal{F}}\left(\left\{x \in X: C \lambda^{-1} \mathcal{M}^{\gamma / C, *} u(x)>1\right\}\right) \\
& \leq C \lambda^{-p}\left\|\mathcal{M}^{\gamma / C, *} u\right\|_{\mathcal{F}(X)}^{p} \\
& \leq C \lambda^{-p}\|u\|_{\mathcal{F}(X)}^{p},
\end{aligned}
$$

and the claim follows.

Proof of Theorem 1.2. We assume first that $u \in \mathcal{F}(X)$. The general case, $u \in \dot{\mathcal{F}}(X)$, then follows by a localization argument.

We have to show that the $\operatorname{limit}_{\lim _{r \rightarrow 0}} m_{u}^{\gamma}(B(x, r))=u^{*}(x)$ exists outside a set of zero $\mathcal{F}$-capacity and that $u^{*}$ is an $\mathcal{F}$-quasicontinuous representative of $u$.

By Theorem 1.1, if $0<q<\infty$, and by [41, Proposition 4.5], if $q=\infty$, there are continuous functions $u_{i}, i \in \mathbb{N}$, such that

$$
\left\|u-u_{i}\right\|_{\mathcal{F}(X)}^{p}<2^{-(1+p) i}
$$

for each $i \in \mathbb{N}$.

For each $n, i, k \in \mathbb{N}$, let

$$
A_{n, i}=\left\{x: \mathcal{M}^{1 /(2 n)}\left(u-u_{i}\right)(x)>2^{-i}\right\} \quad \text { and } \quad B_{n, k}=\bigcup_{i \geq k} A_{n, i} .
$$


We will show that the set

$$
E=\bigcup_{n \in \mathbb{N}} E_{n}
$$

where

$$
E_{n}=\bigcap_{k \in \mathbb{N}} B_{n, k}
$$

is of zero capacity and that the limit of medians of $u$ exists outside $E$.

By Theorem [7.7, the capacity of each set $A_{n, i}$ has an upper bound,

$$
C_{\mathcal{F}}\left(A_{n, i}\right) \leq C 2^{i p}\left\|u-u_{i}\right\|_{\mathcal{F}(X)}^{p}<C 2^{-i}
$$

and hence, by (6.1),

$$
C_{\mathcal{F}}\left(B_{n, k}\right) \leq C 2^{-k}
$$

This implies that $C_{\mathcal{F}}(E)=0$.

Next we show that the continuous approximations $u_{i}$ of $u$ converge uniformly to a continuous function outside $B_{n, k}$ and that the limit function is the limit of $\gamma$-medians of $u$. Let $1 / n \leq \gamma \leq 1 / 2$. By Lemma 2.7, we have

$$
\begin{aligned}
\left|u_{i}(x)-m_{u}^{\gamma}(B(x, r))\right| & =\left|m_{\left(u-u_{i}(x)\right)}^{\gamma}(B(x, r))\right| \leq m_{\left|u-u_{i}(x)\right|}^{1 / n}(B(x, r)) \\
& \leq m_{\left|u-u_{i}\right|}^{1 /(2 n)}(B(x, r))+m_{\left|u_{i}-u_{i}(x)\right|}^{1 /(2 n)}(B(x, r)),
\end{aligned}
$$

which, together with the fact that $m_{\left|u_{i}-u_{i}(x)\right|}^{1 /(2 n)}(B(x, r)) \rightarrow 0$ as $r \rightarrow 0$ by the continuity of $u_{i}$, implies that for each $x \in X \backslash A_{n, i}$,

$$
\limsup _{r \rightarrow 0}\left|u_{i}(x)-m_{u}^{\gamma}(B(x, r))\right| \leq \mathcal{M}^{1 /(2 n)}\left(u-u_{i}\right)(x) \leq 2^{-i} .
$$

Now, for each $x \in X \backslash B_{n, k}$ and $i, j \geq k$, we have

$$
\begin{aligned}
\left|u_{i}(x)-u_{j}(x)\right| & \leq \limsup _{r \rightarrow 0}\left(\left|u_{i}(x)-m_{u}^{\gamma}(B(x, r))\right|+\left|u_{j}(x)-m_{u}^{\gamma}(B(x, r))\right|\right) \\
& \leq 2^{-i}+2^{-j}
\end{aligned}
$$

which implies that $\left(u_{i}\right)$ converges uniformly in $X \backslash B_{n, k}$ to a continuous function $v$. Since

$$
\limsup _{r \rightarrow 0}\left|v(x)-m_{u}^{\gamma}(B(x, r))\right| \leq\left|v(x)-u_{i}(x)\right|+\limsup _{r \rightarrow 0}\left|u_{i}(x)-m_{u}^{\gamma}(B(x, r))\right|,
$$

it follows that

$$
v(x)=\lim _{r \rightarrow 0} m_{u}^{\gamma}(B(x, r))=u^{*}(x)
$$

for every $x \in X \backslash B_{n, k}$ and $1 / n \leq \gamma \leq 1 / 2$. Hence the limit

$$
\lim _{r \rightarrow 0} m_{u}^{\gamma}(B(x, r))=u^{*}(x)
$$

exist for every $x \in X \backslash E$ and $0<\gamma \leq 1 / 2$.

Now, assume that $u \in \dot{\mathcal{F}}(X)$. Let $x_{0} \in X$ and, for each $k \in \mathbb{N}$, let $\varphi_{k}$ be a Lipschitz function such that $\varphi=1$ on $B\left(x_{0}, k\right)$ and $\varphi=0$ in $X \backslash B\left(x_{0}, 2 k\right)$. Then, 
by [20, Lemma 3.10 and Remark 3.11], $u_{k}=u \varphi_{k} \in \mathcal{F}(X)$. By the first part of the proof, for every $k$, there exists a set $E_{k}$ with $C_{\mathcal{F}}\left(E_{k}\right)=0$ such that the limit

$$
\lim _{r \rightarrow 0} m_{u_{k}}^{\gamma}(B(x, r))=u_{k}^{*}(x)
$$

exists for every $x \in X \backslash E_{k}$ and $0<\gamma \leq 1 / 2$. Since

$$
\lim _{r \rightarrow 0} m_{u}^{\gamma}(B(x, r))=\lim _{r \rightarrow 0} m_{u_{k}}^{\gamma}(B(x, r))
$$

in $B\left(x_{0}, k\right)$, it follows that the limit

$$
\lim _{r \rightarrow 0} m_{u}^{\gamma}(B(x, r))=u^{*}(x)
$$

exists for every $x \in X \backslash \cup_{k \in \mathbb{N}} E_{k}$. By (6.1), $C_{\mathcal{F}}\left(\cup_{k \in \mathbb{N}} E_{k}\right)=0$.

Let $\varepsilon>0$. By the first part of the proof, for every $k$, there exists a set $U_{k}$ with $C_{\mathcal{F}}\left(U_{k}\right)<2^{-k} \varepsilon$ such that $u_{k}^{*}$ is continuous in $X \backslash U_{k}$. Since $u^{*}=u_{k}^{*}$ in $B\left(x_{0}, k\right)$, it follows that $u^{*}$ is continuous in $X \backslash \cup_{k \in \mathbb{N}} U_{k}$. By (6.1),$C_{\mathcal{F}}\left(\cup_{k \in \mathbb{N}} U_{k}\right)<C \varepsilon$. Thus, $u^{*}$ is $\mathcal{F}$-quasicontinuous.

\section{LeBESGUE POINTS}

For restricted values of $p$ and $q$, counterparts of Theorems [1.2, 77.6 and 7.7 hold also for integral averages. The main result of this section is the following.

Theorem 8.1. Let $\mathcal{F}=N_{p, q}^{s}$, where $0<s<1, Q /(Q+s)<p<\infty, 0<q<\infty$ or $\mathcal{F}=M_{p, q}^{s}$, where $0<s<1$ and $Q /(Q+s)<p, q<\infty$ or $0<s \leq 1$, $Q /(Q+s)<p<\infty$ and $q=\infty$. If $u \in \dot{\mathcal{F}}(X)$, then $\mathcal{F}$-quasievery point is a Lebesgue point of $u$. Moreover, $u^{*}(x)=\lim _{r \rightarrow 0} u_{B(x, r)}$ is an $\mathcal{F}$-quasicontinuous representative of $u$.

Throughout the rest of this section, we assume that $\mathcal{F}$ satisfies the assumptions of Theorem 8.1. Since the condition $p>Q /(Q+s)$ guarantees only local integrability of Besov or Triebel-Lizorkin functions, we have to use restricted maximal functions

$$
\mathcal{M}_{R} u(x)=\sup _{0<r<R}|u|_{B(x, r)}
$$

and

$$
\mathcal{M}_{R}^{*} u(x)=\sup _{2^{k}<R}|u|_{2^{k}}(x) .
$$

Notice that if $u$ is locally integrable and $R<\infty$, then $\mathcal{M}_{R} u$ and $\mathcal{M}_{R}^{*} u$ are almost everywhere finite. It is easy to see that there exists a constant $C=C\left(c_{d}\right)$ such that

$$
C^{-1} \mathcal{M}_{C^{-1} R} u \leq \mathcal{M}_{R}^{*} u \leq C \mathcal{M}_{C R} u \text {. }
$$

Theorem 8.2 below follows from the proofs of Theorems 3.4, 4.7 and 4.8 in [21].

Theorem 8.2. Let $0<R<\infty$. Then there exists a constant $C=C\left(c_{d}, s, p, q\right)$ such that

for all $u \in \dot{\mathcal{F}}(X)$.

$$
\left\|\mathcal{M}_{R}^{*} u\right\|_{\dot{\mathcal{F}}(X)} \leq C\|u\|_{\dot{\mathcal{F}}(X)}
$$

We need the following simple lemma. 
Lemma 8.3. Suppose that $u=0$ outside $B=B\left(x_{0}, r\right)$ and that there exists $y_{0} \in X$ such that $d\left(x_{0}, y_{0}\right)=3 r$. Then there is a constant $C=C\left(c_{d}, s, p, q\right)$ such that $\|u\|_{\mathcal{F}(X)} \leq C\left(1+r^{s}\right)\|u\|_{\dot{\mathcal{F}}(X)}$.

Proof. Let $\left(g_{k}\right)_{k \in \mathbb{Z}} \in \mathbb{D}^{s}(u)$. Then, for almost every $x \in B$,

$$
|u(x)| \leq 5^{s} r^{s}(g(x)+\underset{y \in 4 B \backslash 2 B}{\operatorname{essinf}} g(y)),
$$

where $g=\max \left\{g_{k}: r / 2 \leq 2^{-k}<10 r\right\}$. Since $B\left(y_{0}, r\right) \subset 4 B \backslash 2 B$ and $B \subset B\left(y_{0}, 4 r\right)$, it follows from the doubling property that $\mu(B) \leq C \mu(4 B \backslash 2 B)$. Hence

$$
\begin{aligned}
\|u\|_{L^{p}(X)} & =\|u\|_{L^{p}(B)} \leq C r^{s}\|g\|_{L^{p}(4 B)} \\
& \leq C r^{s} \min \left\{\left\|\left(g_{k}\right)\right\|_{l^{q}\left(L^{p}(X)\right)},\left\|\left(g_{k}\right)\right\|_{L^{p}\left(X, l^{q}\right)}\right\} .
\end{aligned}
$$

Since this holds for every $\left(g_{k}\right)_{k \in \mathbb{Z}} \in \mathbb{D}^{s}(u)$, we have that

$$
\|u\|_{L^{p}(X)} \leq C r^{s}\|u\|_{\dot{\mathcal{F}}(X)}
$$

and the claim follows.

By combining Theorem 8.2 and Lemma 8.3 , we obtain a localized capacitary weak-type estimate for the restricted Hardy-Littlewood maximal function.

Theorem 8.4. Let $B=B\left(x_{0}, r\right)$ be a ball and assume that the sphere $\left\{y: d\left(y, x_{0}\right)=\right.$ $30 r\}$ is nonempty. Then there exist constants $c=c\left(c_{d}\right)$ and $C=C\left(c_{d}, s, p, q, r\right)$ such that

$$
C_{\mathcal{F}}\left(\left\{x \in B: \mathcal{M}_{r / c} u(x)>\lambda\right\}\right) \leq C \lambda^{-p}\|u\|_{\mathcal{F}(X)}^{p}
$$

for all $u \in \mathcal{F}(X)$.

Proof. Let $c$ be the constant in (8.1). Then $\mathcal{M}_{r / c} u(x) \leq c \mathcal{M}_{r}^{*} u(x)$. Let $\varphi \geq 0$ be a Lipschitz function such that $\left.\varphi\right|_{5 B}=1$ and $\left.\varphi\right|_{X \backslash 6 B}=0$. Then, by [20, Lemma 3.10 and Remark 3.11], $u \varphi \in \mathcal{F}(X)$ and

$$
\|u \varphi\|_{\mathcal{F}(X)} \leq C\|u\|_{\mathcal{F}(X)} .
$$

If $x \in B$, then $\mathcal{M}_{r}^{*} u(x)=\mathcal{M}_{r}^{*}(u \varphi)(x)$. Hence,

$$
\left\{x \in B: \mathcal{M}_{r / c} u(x)>\lambda\right\} \subset\left\{x \in X: c \lambda^{-1} \mathcal{M}_{r}^{*}(u \varphi)(x)>1\right\}
$$

and so

$$
C_{\mathcal{F}}\left(\left\{x \in B: \mathcal{M}_{r / c} u(x)>\lambda\right\}\right) \leq C \lambda^{-p}\left\|\mathcal{M}_{r}^{*}(u \varphi)\right\|_{\mathcal{F}(X)}^{p} .
$$

If $x \in X \backslash 10 B$, then $\mathcal{M}_{r}^{*}(u \varphi)(x)=0$. Thus, by Lemma 8.3, Theorem 8.2 and (8.2),

$$
\left\|\mathcal{M}_{r}^{*}(u \varphi)\right\|_{\mathcal{F}(X)} \leq C\left\|\mathcal{M}_{r}^{*}(u \varphi)\right\|_{\dot{\mathcal{F}}(X)} \leq C\|u \varphi\|_{\dot{\mathcal{F}}(X)} \leq C\|u\|_{\mathcal{F}(X)}
$$

and the claim follows.

Proof of Theorem 8.1. We may assume that $X$ contains at least two points. Then $X$ can be covered by balls $B_{i}=B\left(x_{i}, r_{i}\right), i \in I$, where $I \subset \mathbb{N}$, such that the spheres $\left\{y: d\left(x_{i}, y\right)=30 r_{i}\right\}$ are nonempty.

Theorem 8.4 and a similar argument as in the proof of Theorem 1.2 imply that, for every $i$, there exists a set $E_{i}$ with $C_{\mathcal{F}}\left(E_{i}\right)=0$ such that the limit $\lim _{r \rightarrow 0} u_{B(x, r)}=$ 
$u^{*}(x)$ exists in $B_{i} \backslash E_{i}$. Moreover, for every $\varepsilon>0$, there exists a set $U_{i}$ such that $C_{\mathcal{F}}\left(U_{i}\right)<2^{-i} \varepsilon$ and $u^{*}$ is continuous in $B_{i} \backslash U_{i}$. Hence, the limit

$$
\lim _{r \rightarrow 0} u_{B(x, r)}=u^{*}(x)
$$

exists in $X \backslash \cup_{i \in I} E_{i}$ and $u^{*}$ is continuous in $X \backslash \cup_{i \in I} U_{i}$. By 6.1), $C_{\mathcal{F}}\left(\cup_{i \in I} E_{i}\right)=0$ and $C_{\mathcal{F}}\left(\cup_{i \in I} U_{i}\right)<C \varepsilon$.

The following corollary of Theorem 8.1 extends the results obtained in [26], [28] and [39] to the case $Q /(Q+s)<p<1$.

Corollary 8.5. Let $0<s \leq 1, Q /(Q+s)<p<\infty$ and $u \in \dot{M}^{s, p}(X)$. Then $M^{s, p}$-quasievery point is a Lebesgue point of $u$ and $u^{*}(x)=\lim _{r \rightarrow 0} u_{B(x, r)}$ is an $M^{s, p}$-quasicontinuous representative of $u$.

Acknowledgements: The research was supported by the Academy of Finland. Part of this research was conducted during the visit of the third author to Forschungsinstitut für Mathematik of ETH Zürich, and she wishes to thank the institute for the kind hospitality.

\section{REFERENCES}

[1] D. R. Adams: The classification problem for the capacities associated with the Besov and Triebel-Lizorkin spaces, Approximation and function spaces (Warsaw, 1986), 9-24, Banach Center Publ., 22, PWN, Warsaw, 1989.

[2] D. R. Adams: Besov capacity redux, Problems in mathematical analysis. No. 42. J. Math. Sci. (N. Y.) 162 (2009), no. 3, 307-318.

[3] D. R. Adams and L. I. Hedberg: Function spaces and potential theory, Grundlehren der Mathematischen Wissenschaften, 314. Springer-Verlag, Berlin, 1996.

[4] D. R. Adams and R. Hurri-Syrjänen: Besov functions and vanishing exponential integrability, Illinois J. Math. 47 (2003), no. 4, 1137-1150.

[5] D. R. Adams and J. Xiao: Strong type estimates for homogeneous Besov capacities, Math. Ann. 325 (2003), no. 4, 695-709.

[6] M. Bonk, E. Saksman and T. Soto: Triebel-Lizorkin spaces on metric spaces via hyperbolic fillings, preprint 2014, arXiv:1411.5906.

[7] M. Bourdon: Une caractérisation algébrique des homéomorphismes quasi-Möbius, Ann. Acad. Sci. Fenn. Math. 32 (2007), no. 1, 235-250.

[8] R. R Coifman and G. Weiss: Analyse harmonique non-commutative sur certains espaces homogénes, Lecture Notes in Mathematics, Vol.242. Springer-Verlag, Berlin-New York, 1971.

[9] Ş. Costea: Besov capacity and Hausdorff measures in metric measure spaces, Publ. Mat. 53 (2009), no. 1, 141-178.

[10] J. R. Dorronsoro, On the differentiability of Lipschitz-Besov functions, Trans. Amer. Math. Soc. 303 (1987), 229-240.

[11] L.C. Evans and R.F. Gariepy: Measure Theory and Fine Properties of Functions, CRC Press, 1992, Boca Raton-New York-London-Tokyo.

[12] H. Federer and W.P. Ziemer: The Lebesgue set of a function whose distribution derivatives are $p$-th power summable, Indiana Univ. Math. J. 22 (1972), 139-158.

[13] C. Fefferman and E. M. Stein: Some maximal inequalities, Amer. J. Math. 93 (1971), 107115.

[14] N. Fujii: A condition for a two-weight norm inequality for singular integral operators, Studia Math. 98 (1991), no. 3, 175-190. 
[15] A. Gogatishvili, P. Koskela and Y. Zhou: Characterizations of Besov and Triebel-Lizorkin Spaces on Metric Measure Spaces, Forum Math. 25 (2013), no. 4, 787-819.

[16] L. Grafakos, L. Liu and D. Yang: Vector-valued singular integrals and maximal functions on spaces of homogeneous type, Math. Scand. 104 (2009), 296-310.

[17] P. Hajłasz: Sobolev spaces on an arbitrary metric space, Potential Anal. 5 (1996), 403-415.

[18] Y. Han, D. Müller, and D. Yang: A theory of Besov and Triebel-Lizorkin spaces on metric measure spaces modeled on Carnot-Carathéodory spaces, Abstr. Appl. Anal. 2008, Art. ID $893409,250 \mathrm{pp}$.

[19] L. I. Hedberg and Y. Netrusov: An axiomatic approach to function spaces, spectral synthesis, and Luzin approximation, Mem. Amer. Math. Soc. 188 (2007), no. 882

[20] T. Heikkinen, L. Ihnatsyeva and H. Tuominen: Measure density and extension of Besov and Triebel-Lizorkin functions, to appear in J. Fourier Anal. Appl., http://arxiv.org/abs/1409.0379

[21] T. Heikkinen and H. Tuominen: Smoothing properties of the discrete fractional maximal operator on Besov and Triebel-Lizorkin spaces, Publ. Mat. 58 (2014), no. 2. 379-399.

[22] T. Heikkinen and H. Tuominen: Approximation by Hölder functions in Besov and TriebelLizorkin spaces, preprint 2015, http://arxiv.org/abs/1504.02585

[23] B. Jawerth, C. Perez, and G. Welland: The positive cone in Triebel-Lizorkin spaces and the relation among potential and maximal operators, Harmonic analysis and partial differential equations (Boca Raton, FL, 1988), 71-91, Contemp. Math., 107, Amer. Math. Soc., Providence, RI, 1990.

[24] B. Jawerth and A. Torchinsky: Local sharp maximal functions, J. Approx. Theory 43 (1985), no. $3,231-270$.

[25] F. John: Quasi-isometric mappings, Seminari 1962/63 Anal. Alg. Geom. e Topol., vol. 2, Ist. Naz. Alta Mat., Ediz. Cremonese, Rome, 1965, pp. 462-473.

[26] J. Kinnunen and V. Latvala: Lebesgue points for Sobolev functions on metric spaces, Rev. Mat. Iberoamericana 18 (2002), no. 3, 685-700.

[27] J. Kinnunen and O. Martio: The Sobolev capacity on metric spaces, Ann. Acad. Sci. Fenn. Math. 21 (1996), 367-382.

[28] J. Kinnunen and H. Tuominen: Pointwise behaviour of $M^{1,1}$ Sobolev functions, Math. Z. 257 (2007), no. 3, 613-630.

[29] P. Koskela and E. Saksman: Pointwise characterizations of Hardy-Sobolev functions, Math. Res. Lett. 15 (2008), no. 4, 727-744.

[30] P. Koskela, D. Yang and Y. Zhou: Pointwise Characterizations of Besov and Triebel-Lizorkin Spaces and Quasiconformal Mappings, Adv. Math. 226 (2011), no. 4, 3579-3621.

[31] A. K. Lerner, A pointwise estimate for the local sharp maximal function with applications to singular integrals, Bull. London Math. Soc. 42 (2010), 843-856.

[32] A. K. Lerner and C. Pérez: Self-improving properties of generalized Poincaré type inequalities throught rearrangements, Math. Scand. 97 (2) (2005), 217-234.

[33] R. A. Macías and C. Segovia: A decomposition into atoms of distributions on spaces of homogeneous type, Adv. in Math. 33 (1979), no. 3, 271-309.

[34] Y. V. Netrusov: Sets of singularities of functions in spaces of Besov and Lizorkin-Triebel type. (Russian), Trudy Mat. Inst. Steklov. 187 (1989), 162-177. translation in Proc. Steklov Inst. Math. 1990, no. 3, 185-203.

[35] Y. V. Netrusov: Metric estimates for the capacities of sets in Besov spaces. (Russian), Trudy Mat. Inst. Steklov. 190 (1989), 159-185. translation in Proc. Steklov Inst. Math. 1992, no. $1,167-192$.

[36] Y. V. Netrusov: Estimates of capacities associated with Besov spaces. (Russian), Zap. Nauchn. Sem. S.-Peterburg. Otdel. Mat. Inst. Steklov. (POMI) 201 (1992), 124-156, translation in J. Math. Sci. 78 (1996), no. 2, 199-217.

[37] C. J. Neugebauer, Strong differentiability of Lipschitz functions, Trans. Amer. Math. Soc. 240 (1978), 295-306. 
[38] J. Poelhuis and A. Torchinsky: Medians, continuity, and vanishing oscillation, Studia Math. 213 (2012), no. 3, 227-242.

[39] M.A. Prohorovich: Hausdorff dimension of Lebesgue sets for $W_{\alpha}^{p}$ classes on metric spaces, Matematicheskie Zametki 2007. V.82, Number 1. P.99-107. (in Russian, English translation in Math. Notes, 2007, V. 82, no.1)

[40] Y. Sawano: Sharp estimates of the modified Hardy-Littlewood maximal operator on the nonhomogeneous space via covering lemmas, Hokkaido Math. J. 34 (2005), no. 2, 435-458.

[41] N. Shanmugalingam, D. Yang, and W. Yuan: Newton-Besov Spaces and NewtonTriebel-Lizorkin Spaces on Metric Measure Spaces, to appear in Positivity, http://dx.doi.org/10.1007/s11117-014-0291-7

[42] B. Stocke, Differentiability properties of Bessel potentials and Besov functions, Ark. Math. 22 (1984), 269-286.

[43] J-O. Strömberg: Bounded mean oscillation with Orlicz norms and duality of Hardy spaces, Indiana Univ. Math. J. 28 (1979), no. 3, 511-544.

[44] H. Triebel: Theory of Function Spaces, Birkhäuser Verlag, Basel, 1983.

[45] D. Yang: New characterizations of Hajłasz-Sobolev spaces on metric spaces, Sci. China Ser. A 46 (2003), 675-689.

[46] Y. Zhou: Fractional Sobolev extension and imbedding, Trans. Amer. Math. Soc. 367 (2015), no. 2, 959-979.

[47] W. P. Ziemer: Weakly differentiable functions, Graduate Texts in Mathematics, 120, Springer-Verlag, 1989.

T.H., Department of Mathematics, P.O. Box 11100, FI-00076 Aalto UniverSITY, FINLAND

toni.heikkinen@aalto.fi

P.K., Department of Mathematics and Statistics, P.O. Box 35, FI-40014 UniVERSITY OF JYVÄSKYLÄ, FINLAND

pekka.j.koskela@jyu.fi

H.T., Department of Mathematics and Statistics, P.O. Box 35, FI-40014 UniVERSITY OF JYVÄSKYLÄ, FINLAND

heli.m.tuominen@jyu.fi 